Published in final edited form as:

J Med Chem. 2017 March 23; 60(6): 2439-2455. doi:10.1021/acs.jmedchem.6b01825.

\title{
Inhibition of the Aldehyde Dehydrogenase 1/2 Family by Psoralen and Coumarin Derivatives
}

\author{
Cameron D. Buchman and Thomas D. Hurley ${ }^{\star}$ \\ Department of Biochemistry and Molecular Biology, Indiana University School of Medicine, \\ Indianapolis, Indiana 46202, United States
}

\begin{abstract}
Aldehyde dehydrogenase 2 (ALDH2), one of 19 ALDH superfamily members, catalyzes the $\mathrm{NAD}^{+}$-dependent oxidation of aldehydes to their respective carboxylic acids. In this study, we further characterized the inhibition of four psoralen and coumarin derivatives towards ALDH2 and compared them to the ALDH2 inhibitor daidzin for selectivity against five ALDH1/2 isoenzymes. Compound $2\left(\mathrm{~K}_{\mathrm{i}}=19 \mathrm{nM}\right)$ binds within the aldehyde-binding site of the free enzyme species of ALDH2. Thirty-three structural analogs were examined to develop a stronger SAR profile. Seven compounds maintained or improved upon the selectivity towards one of the five ALDH1/2 isoenzymes, including compound 36, a selective inhibitor for ALDH2 $\left(\mathrm{K}_{\mathrm{i}}=2.4 \mu \mathrm{M}\right)$ and compound 32, which was 10-fold selective for ALDH1A1 $\left(\mathrm{K}_{\mathrm{i}}=1.2 \mu \mathrm{M}\right)$ versus ALDH1A2. Further medicinal chemistry on the compounds' basic scaffold could enhance the potency and selectivity for ALDH1A1 or ALDH2 and generate chemical probes to examine the unique and overlapping functions of the ALDH1/2 isoenzymes.
\end{abstract}

\section{Graphical Abstract}

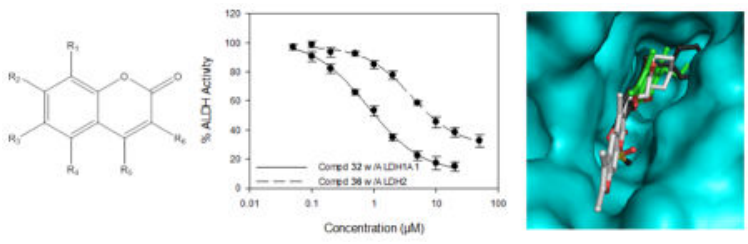

*Corresponding Author: Tel: +1 3172782008 thurley@iu.edu (T.D. Hurley).

AUTHOR CONTRIBUTIONS

These authors contributed equally

\section{ASSESSION CODES}

Authors will release the atomic coordinates and experimental data for the structures of ALDH2 in complex with compound 2 (PDB ID: 5L13) and ALDH1A1 in complex with compound 15 (5L2M), 32 (5L2O), and 34 (5L2N) upon article publication.

SUPPORTING INFORMATION

Compound Purity (PDF)

Molecular formula strings (CSV)

\section{CONFLICT OF INTEREST}

Thomas D. Hurley holds significant financial equity in SAJE Pharma, LLC. However, none of the work described in this study is related to, based on or supported by the company. 


\section{INTRODUCTION}

Aldehydes can lead to cytotoxicity and carcinogenesis when present in large enough quantities within the human body. ${ }^{1,2}$ The human body encounters numerous aldehydes from both the external environment and the internal metabolism of biomolecules. ${ }^{3}$ The aldehyde dehydrogenases (ALDHs) are one of many enzyme systems the body utilizes to alleviate aldehyde stress. ${ }^{4}$ The human genome has 19 functional genetic loci for members of the ALDH superfamily, the majority of which catalyze the $\mathrm{NAD}(\mathrm{P})^{+}$dependent oxidation of aldehydes to their respective carboxylic acids or CoA esters. ${ }^{5,6}$ ALDHs are separated into families and subfamilies based on their sequence similarity. ${ }^{7}$ The 19 ALDHs share similar yet distinct functions due to their varying substrate specificities and gene expression differences. Some are ubiquitously expressed, such as ALDH1A1 and ALDH2, while are other are expressed preferentially in certain tissues or during certain periods of development. Naturally occurring mutations within various ALDHs can cause human diseases such as Sjogren-Larsson syndrome (ALDH3A2), ${ }^{8}$ type II hyperprolinemia (ALDH4A1), ${ }^{9} 4$ hydroxybutyricaciduria (ALDH5A1) ${ }^{10}$, and pyridoxine dependent epilepsy (ALDH7A1). ${ }^{11}$

The ALDH1/2 family consists of ALDH1A1, ALDH1A2, ALDH1A3, ALDH1B1, and ALDH2. The five isoenzymes primarily oxidize aliphatic aldehydes of varying length. ALDH1A1, ALDH1A2, and ALDH1A3 are cytosolic proteins which are involved in retinoid metabolism. ${ }^{12}$ ALDH1A1 has been implicated in providing resistance to certain anti-cancer agents, such as cyclophosphamide, as well as having a functional role in cancer stem cells. ${ }^{13-15}$ ALDH1A2 and ALDH1A3 are critical for embryonic development in mice as individual genetic knockout of these two genes do not produce viable animals. ${ }^{16,17}$ ALDH2 is a mitochondrial enzyme most known for its role in acetaldehyde metabolism during the conversion of ethanol to acetic acid. ${ }^{18}$ Other isoenzymes such as ALDH1A1 and ALDH1B1 also can contribute to the oxidation of acetaldehyde, especially when ALDH2 activity is reduced by the presence of the ALDH2*2 allele. ${ }^{19-21}$ ALDH1B1, a mitochondrial enzyme most similar to ALDH2, has recently been linked with colon cancer and diabetes. ${ }^{22,}{ }^{23}$ ALDH1A1 and ALDH2 have also been linked to the metabolism of dopamine within different areas of the brain. ${ }^{24}$ For the ALDH1/2 family of isoenzymes it is difficult to make specific assignment of function due to their overlapping substrate specificities. The discovery and development of isoenzyme-selective inhibitors or activators would better allow investigators to identify the individual contribution of these individual isoenzymes to the metabolism of these common substrates.

The majority of the ALDH superfamily, including the ALDH1/2 family, shares the same basic catalytic mechanism. $\mathrm{NAD}(\mathrm{P})^{+}$binds to the enzyme and can sample multiple binding positions. ${ }^{25}$ The conserved catalytic cysteine (Cys302 in ALDH2) is activated and performs a nucleophilic attack on the carbonyl carbon of the aldehyde forming a tetrahedral intermediate. ${ }^{26}$ Once $\mathrm{NAD}(\mathrm{P})^{+}$occupies the necessary binding position, the aldehydic hydride ion is transferred to the nicotinamide ring of $\mathrm{NAD}(\mathrm{P})^{+}$forming $\mathrm{NAD}(\mathrm{P}) \mathrm{H}$. The substrate-enzyme complex then undergoes a conformation change in which the $\mathrm{NAD}(\mathrm{P}) \mathrm{H}$ moves away to allow access of the catalytic site to a water molecule. The water molecule is deprotonated by a conserved glutamate residue (Glu268 in ALDH2) and performs a nucleophilic attack on the carbonyl carbon of the acyl-enzyme intermediate. ${ }^{27}$ The bond 
between the sulfur and carbonyl carbon is then broken regenerating the free enzyme and producing the carboxylic acid end product. Due to the similar catalytic mechanisms for the ALDH1/2 family, mechanism-based inhibitors may lack selectivity. However, selectivity can be achieved through taking advantage of the different aldehyde binding sites of the ALDH family which have evolved over time through substitutions of residues in the substrate binding tunnel.

One use of a chemical probe for ALDH 2 is the treatment of alcohol abuse. The ALDH $2 * 2$ allele is found in $\sim 40 \%$ of East Asians and leads to the alcohol flush response. ${ }^{28,} 29$ The E504K substitution (E487K after removal of the mitochondrial targeting signal) in the ALDH $2 * 2$ allele leads to decreased acetaldehyde metabolism to the point where individuals with the allele will develop nausea, vomiting, and vasodilation shortly after alcohol consumption. The ALDH2*2 allele is associated with a lower risk of alcoholism due in part to this physiological response. ${ }^{30}$ Mimicking the ALDH $2 * 2$ response is an established treatment option for alcohol abuse. Disulfiram and daidzin and their derivatives are commonly used to treat alcohol abuse as ALDH2 inhibitors. ${ }^{31}$ However, disulfiram is nonselective and also inhibits ALDH1A1 as disulfiram causes the irreversible inactivation of ALDH enzymes through carbamoylation of the catalytic cysteine residue. ${ }^{32,} 33$ Additionally, disulfiram metabolites will chelate with copper and thereby inhibit copper-dependent enzymes. ${ }^{34,} 35$ Though daidzin reversibly inhibits ALDH2 two orders of magnitude greater than ALDH1A1, its effect on the activity of ALDH1A2, ALDH1A3, and ALDH1B1 has been understudied. 36,37

We sought to further characterize the original set of psoralen and coumarin derivatives discovered in a high-throughput screen and expand upon those hits by examining a series of related analogs as inhibitors of the ALDH1/2 isoenzymes. These compounds are unique from the covalent inactivating Aldi compounds and N-N-diethylaminobenzaldehyde that we and others have previously characterized. ${ }^{38-40}$ Our initial goal was to determine the compounds' mechanism of action and to understand the structural basis for their inhibition of ALDH2, as their inhibition towards ALDH2 was the strongest amongst the ALDH1/2 isoenzymes. In order to create a common basis for comparison in our assays the inhibition profile for daidzin toward these same ALDH1/2 family members was also determined. Thirty-three additional psoralen and coumarin analogs were then evaluated as inhibitors toward the ALDH1/2 family of isoenzymes. Although many of the structural variations resulted in the loss of inhibition towards ALDH2, one coumarin derivative was found to be an ALDH2-selective inhibitor. Surprisingly a different coumarin derivative was found to be an ALDH1A1-selective inhibitor. Both compounds could be further developed to make them more selective for their respective isoenzymes. The overall chemical similarity between the compounds while possessing different isozyme selectivity highlights the effect small chemical changes can have when developing isoenzyme-selective probes for ALDH isoenzymes. 


\section{RESULTS AND DISCUSSION}

\section{Further Characterization of the Four Initial Aromatic Lactones}

Four aromatic lactones were previously identified as inhibitors of the ALDH1/2 family of enzymes, 1 (2CB5), 2 (2P3), 3 (2P4) and 4 (2BS4) (Figure 1). ${ }^{41}$ The $\mathrm{IC}_{50}$ values for daidzin for the ALDH1/2 family were measured to compare our results to compounds (including CVT-10216) already described in the literature as ALDH2-selective inhibitors. ${ }^{36,} 37,42$ Consistent with prior work, daidzin was found to be about 100-fold more potent toward ALDH2 than ALDH1A1 (Table 1). However, our $\mathrm{IC}_{50}$ values are 10-fold higher than prior values. The discrepancy between the values reported here and the values in the literature stems from the fact that our group uses propionaldehyde ( $\mathrm{K}_{\mathrm{m}}$ for ALDH2 is $\sim 0.1 \mu \mathrm{M}$ ) as the standard substrate for the ALDH1/2 isoenzymes at $100 \mu \mathrm{M}$ and the original reports on isoflavones, such as daidzin and prunetin, utilized formaldehyde ( $\left(\mathrm{K}_{\mathrm{m}}\right.$ for ALDH2 is $\sim 320$ $\mu \mathrm{M})^{43}$ as the substrate at $600 \mu \mathrm{M} .{ }^{36}$ We chose to utilize propionaldehyde, rather than formaldehyde, as substrate in our assays in order to have as many enzymes as possible evaluated for inhibition against a single common substrate (propionaldehyde was used in all enzyme assays, except ALDH3A1). On the other hand, because $\mathrm{IC}_{50}$ measurements are dependent on the concentration utilized in the assays relative to their isoenzyme specific $\mathrm{K}_{\mathrm{m}}$ values, our assays will be more stringent for inhibition, because only very strong inhibitors will be able to overcome the 1,000-fold concentration excess of propionaldehyde in the ALDH2 assay. In regards to the other ALDH1/2 enzymes, we found daidzin to inhibit ALDH1B1 $\left(\mathrm{IC}_{50}=5.1 \pm 0.5 \mu \mathrm{M}\right)$ and ALDH1A2 $\left(\mathrm{IC}_{50}=4.5 \pm 0.6 \mu \mathrm{M}\right)$ with similar potencies to ALDH2 $\left(\mathrm{IC}_{50}=3.5 \pm 0.1 \mu \mathrm{M}\right)$, while being less potent toward ALDH1A1 and ALDH1A3. Inhibition of ALDH1A1 and ALDH1A3 by daidzin was observed, but compound solubility issues prevented full dose-response analyses.

Compounds 1, 2, and $\mathbf{3}$ are psoralen derivatives with varying alkyl substitutions to the aromatic ring structure (Figure 1). Compound $\mathbf{4}$ is a coumarin derivative with a methyl 2methoxypropanoate chain and is a potential substrate for the in vitro esterase reaction of $\mathrm{ALDH} 2$. The $\mathrm{IC}_{50}$ values of the four aromatic lactones for seven isoenzymes were determined in order to better characterize the selectivity of the compounds towards/for the ALDH1/2 family of isoenzymes versus other ALDH isoenzymes as previously only single point activity measurements had been utilized.

The four lactones lacked inhibitory effect toward ALDH1L1 (rat), ALDH3A1, ALDH4A1 and ALDH5A1 ${ }^{41} 4$ showed selectivity towards ALDH2 versus the other eight isoenzymes tested with an $\mathrm{IC}_{50}$ value of $1.5 \pm 0.3 \mu \mathrm{M}$. The other three compounds inhibited ALDH2 most strongly, though they also inhibited the majority of the other ALDH1/2 isoenzymes at sub-micromolar concentrations. The lowest $\mathrm{IC}_{50}$ measured was that of $\mathbf{2}$ for $\mathrm{ALDH} 2\left(\mathrm{IC}_{50}=\right.$ $0.11 \pm 0.02 \mu \mathrm{M}) .2$ inhibited each of the five ALDH1/2 isoenzymes the strongest and was the only one of the three which completely inhibited all five isoenzymes. 3 has similar $\mathrm{IC}_{50}$ values as 2 for ALDH2 $\left(\mathrm{IC}_{50}=0.19 \pm 0.01 \mu \mathrm{M}\right)$ and ALDH1A3, but only partially inhibits ALDH1B1 and ALDH1A2 and does not inhibit ALDH1A1. 1 had the highest $\mathrm{IC}_{50}$ values of the aromatic lactones for the five ALDH1/2 isoenzymes and only partially inhibited ALDH1A1. The partial inhibition of the enzymes by these compounds could not be 
determined from the single point activity measurements previously reported. ${ }^{41}$ The number and length of the alkyl substituents on the aromatic lactones positively correlates with the potency of inhibition towards ALDH1A1 (Figure 1 and Table 1). Covariation experiments were completed with $\mathbf{2}, \mathbf{3}$, and $\mathbf{4}$ to better understand their mechanism of inhibition toward ALDH2 (Figure 2A-C). 2, 3, and 4 were all found to be competitive versus varied $\mathrm{NAD}^{+}$for ALDH2, with $\mathrm{K}_{\mathrm{i}}$ values of $19 \pm 1 \mathrm{nM}, 87 \pm 8 \mathrm{nM}$ and $310 \pm 36 \mathrm{nM}$ respectively. In prior work, 3 was found to be non-competitive (mixed-type) with respect to varied propionaldehyde for ALDH2 with a $\mathrm{K}_{\mathrm{i}}=35 \mathrm{nM}{ }^{41}$ These mechanisms of inhibition were surprising, since the esterase screen utilized to discover these compounds was designed to select against compounds competitive toward coenzyme binding. ${ }^{44}$ Therefore, we solved the crystal structure of $\mathbf{2}$ bound to ALDH2. Compound $\mathbf{2}$ was chosen for this analysis, as it is the largest of the three psoralen derivatives, fully inhibited all ALDH1/2 isoenzymes, and had the lowest $\mathrm{K}_{\mathrm{i}}$ value. The structure of ALDH2 in complex with compound $\mathbf{2}$ was solved to a resolution of $2.40 \AA$, with the ligand modeled at full occupancy in each of the eight monomers (Table 2). In the absence of coenzyme, 2 binds within the substrate binding site of ALDH2 (Figure 2D) and is surrounded by four phenylalanine side chains (residues 170, 296, 459, and 465). To accommodate the binding of $\mathbf{2}$, the catalytic Cys302 rotates towards the $\mathrm{NAD}^{+}$binding site. The propyl alkyl substituent extends into a pocket formed in part by Glu268, Glu476, Trp177, and Thr244 while the methyl substituents on the furan ring are oriented towards the solvent exposed entrance. Given the larger binding site of ALDH1A1, it is possible the additional contacts provided by the extension of the propyl chain into this pocket are essential for ALDH1A1 inhibition. The narrower binding sites for the other ALDH1/2 members apparently do not require these contacts. The lactone carbonyl of 2 mimics the position of a potential aldehyde substrate and hydrogen bonds with the peptide nitrogen of Cys302 (Figure 2E) in a manner similar to that predicted for the incoming aldehyde substrate as it engages the oxyanion hole. In addition to the contribution from hydrophobic interactions, this one hydrogen bond orients the compound in the substrate binding site.

The shift of Cys302 towards the $\mathrm{NAD}^{+}$site likely explains the competitive inhibition pattern towards varied $\mathrm{NAD}^{+}$, since both NAD+ and $\mathbf{2}$ bind to the same enzyme species in solution the free enzyme. A structural comparison of $\mathbf{2}$ and productive NAD $(\mathrm{H})$ binding to ALDH2 demonstrates the impact of Cys302 movement on coenzyme binding (Figure 3). When Cys302 resides in this shifted position, $\mathrm{NAD}^{+}$cannot be productively positioned to accept the hydride ion during the dehydrogenase reaction. Additionally the propyl chain proximal to the aromatic lactone may also hinder the positioning of the nicotinamide ring of $\mathrm{NAD}^{+}$. Similarly, 2 cannot bind to the free enzyme form of ALDH2 if Cys302 is not rotated away. Consequently, the binding of $\mathbf{2}$ prevents the productive binding of $\mathrm{NAD}^{+}$and vice versa. Although the majority of $\mathbf{2}$ binds within the substrate binding site, just enough of the compound overlaps with the nicotinamide binding pocket to explain the competitive inhibition pattern toward $\mathrm{NAD}^{+}$. Compound 2 prevents the productive positioning of $\mathrm{NAD}^{+}$ within the enzyme for catalysis. However, the binding of $\mathrm{NAD}^{+}$in a manner not conducive to catalysis is still possible in the presence of 2 . This is further supported by the inhibition of ALDH 2 by 2 under saturating $\mathrm{NAD}^{+}$conditions for the covariation experiment with propionaldehyde. ${ }^{41}$ The non-competitive (mixed-type) inhibition pattern towards substrate 
could also be explained by non-productive binding of $\mathrm{NAD}^{+}$to the enzyme in the presence of inhibitor. For most linear aliphatic substrates ALDH2 appears to follow an iso-ordered bibi reaction mechanism where $\mathrm{NAD}^{+}$will bind to the enzyme first and the coenzyme undergoes conformational changes prior to assuming the productive conformation required for hydride transfer. ${ }^{45,46}$ When propionaldehyde is varied under saturating $\mathrm{NAD}^{+}$ concentrations, the binding of $\mathrm{NAD}^{+}$to the free enzyme would be essentially irreversible and reduce the population of free enzyme in solution to essentially zero. However, 2 may still bind to one of the non-productively positioned complexes with NAD ${ }^{+}$, such off-pathway complexes would be consistent with the observed kinetic data. ${ }^{47,48}$

As previously discussed, ${ }^{41} \mathbf{4}$ may inhibit ALDH2 through multiple mechanisms, including both through positioning of the aromatic lactone moiety near Cys302, but also through its ester group as a potential competitive esterase substrate. Using the structure of $\mathbf{2}$ bound to $\mathrm{ALDH} 2$ as a basis for interpretation, either the aromatic lactone or the ester could face Cys302 when binding in the substrate binding pocket. Additional experiments demonstrate that $\mathbf{4}$ exhibits a time-dependence for inhibition, but the time-dependence is not observed for the inhibition phase, but is related to the length of time the enzyme remains inhibited after complex formation. After more than a day of incubation with stoichiometric amounts of 4 and ALDH2 the amount of inhibition begins to decrease, suggesting a slow hydrolysis of the ester over time. However, we have not been able to determine the contribution of ALDH2 to ester hydrolysis versus the natural non-enzymatic hydrolysis of the ester in solution, since they have similar time-frames and the free acid form of $\mathbf{4}$ is not inhibitory (see below). Clearly though, the hydrolysis of $\mathbf{4}$ limits its use as an ALDH2 inhibitor for in vivo studies.

\section{Initial Characterization of Psoralen- and Coumarin-Derived Analogs}

High-throughput screening assays are fraught with complications in which the initial hit compounds promote protein aggregation or interfere with the screening assay in a manner that resembles inhibition. Consequently, utilization of an orthogonal assay system where the analytical readout is distinct and the development of interpretable SAR on the hits help to guard against pursuing non-specific compounds. We purchased other psoralen- and coumarin-derived analogs to develop SAR on the initial coumarin and psoralen compounds, and to understand the selectivity of the psoralen analogs for ALDH2 so we could take advantage of the selectivity of $\mathbf{4}$ toward ALDH2. Because of the behavior of $\mathbf{4}$, we hypothesized that similar compounds, without the ester group, could reversibly inhibit ALDH2 while maintaining selectivity. A total of 33 compounds were ordered from ChemDiv, ChemBridge, and Sigma-Aldrich as representatives of all the available analogs using the binding location of $\mathbf{2}$ to ALDH2 as a guide for compound selection. Twelve compounds share the psoralen sub-structure of $\mathbf{1 , 2}$, and $\mathbf{3}$ and twenty-one compounds share the coumarin sub-structure of 4 . The effect of $10 \mu \mathrm{M}$ compound on the activity of ALDH1A1, ALDH2, and ALDH3A1 was measured to determine selectivity amongst these three isoenzymes (Tables 3 and 4). The compound scaffold is oriented as seen in Figure 2F.

The first three analogs (5-7) had additional alkyl chains added to the psoralen structure. The addition of a t-butyl group at $\mathrm{R}_{3}$ in $\mathbf{5}$ lowered the inhibition towards ALDH2. The addition of a methyl group to $\mathrm{R}_{1}$ in 6 nearly eliminated inhibition towards ALDH2. Lengthening the 
alkyl chain at $\mathrm{R}_{5}$ to three carbons in 7 maintained inhibition towards ALDH2. Increasing the size of the ring structure of psoralen had differing results. The addition of a cyclohexane to the furan ring in $\mathbf{8}$ lessened inhibition towards ALDH2. Additional rings proximal to the lactone carbonyl (9-11) maintained inhibition towards ALDH2 showing some selectivity against ALDH1A1. The addition of a phenyl group at $\mathrm{R}_{3}$ in $\mathbf{1 3}$ eliminated inhibition towards ALDH1A1 and ALDH2. The addition of a benzyl group at either $R_{5}$ in $\mathbf{1 4}$ or $R_{6}$ in $\mathbf{1 2}$ negatively impacted inhibition of ALDH2, but led to moderate inhibition of ALDH1A1. Compounds 15 and 16 represent a large group of available compounds in which psoralen derivatives are linked to other chemical structures through a 1-(piperidin-1-yl)propan-1-one (15) or N-benzylpropionamide (16) linker at $\mathrm{R}_{6}$. The 1-(piperidin-1-yl)propan-1-one linker

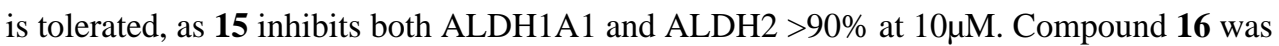
the only psoralen analog which showed inhibition towards ALDH3A1, suggesting the Nbenzylpropionamide linker itself is responsible for the ALDH3A1 inhibition.

Additions to the psoralen scaffold generally resulted in the loss of inhibition towards ALDH2 due to the narrow substrate binding site of ALDH2 (Figure 2F). Adding bulky side chains to the aromatic ring structure reduced or eliminated inhibition of ALDH2 and eventually other ALDH1/2 isoenzymes as seen with the aromatic side chains in 12-14 and the t-Bu group in $\mathbf{5}$. Even smaller groups such as the methyl added to the central aromatic ring in 6 can lead to loss of inhibition. Even though increasing the size of the psoralen derivatives increases the chances of eliminating ALDH2 inhibition, the addition of a fourth ring proximal to the lactone (9-11) maintained ALDH2 selectivity versus ALDH1A1.

The coumarin analogs are variations of $\mathbf{4}$ which all lack the ester group of $\mathbf{4}$. As reported previously, slightly changing the ester portion of $\mathbf{4}$ led to the elimination of ALDH2 inhibition. ${ }^{41}$ Three different methyl ketones (17-19) similar to 4 did not inhibit ALDH2. Additionally 17 and 18 inhibited ALDH1A1 and/or ALDH3A1 unlike 4. An amide (20) similar to 4 inhibited ALDH1A1 as well. Removing the ester side chain to leave the ether (21) led to inhibition of ALDH3A1, while 22, the free acid form of $\mathbf{4}$, did not inhibit any ALDH isoenzymes. These results are consistent with $\mathbf{4}$ behaving differently than the other described analogs and the fact that carboxylates generally are poor inhibitors of ALDH1/2 isoenzymes.

As subtle changes to the methyl 2-methoxypropanoate group of $\mathbf{4}$ at position $\mathrm{R}_{2}$ led to the elimination of ALDH2 inhibition and the loss of selectivity against ALDH1A1 and ALDH3A1, more diverse substituents were examined. The addition of oxygen-containing rings had varied results. A 3-methylfuran ring at $R_{3}$ and $R_{4}$ in $\mathbf{2 3}$ led to partial inhibition of ALDH2, while addition of a furan ring at $R_{1}$ and $R_{2}$ in $\mathbf{2 4}$ led to strong inhibition of ALDH3A1 with no inhibition of ALDH2. The addition of $4 \mathrm{H}$-pyran-4-one at $\mathrm{R}_{2}$ and $\mathrm{R}_{3}$ in 25 led to slight inhibition of ALDH1A1. The addition of a $\delta$-valerolactone at $R_{2}$ and $R_{3}$ in 26 led to inhibition of ALDH2 with lesser inhibition of ALDH1A1 and ALDH3A1. Compounds 27-33 changed the methyl 2-methoxypropanoate group at $R_{2}$ in 4 to more diverse structural groups. In most of these compounds changes at $\mathrm{R}_{2}$ increased inhibition towards ALDH1A1 and ALDH3A1, but diminished inhibition of ALDH2. Compounds 27 and 28 contain unsaturated alkyl chains at $\mathrm{R}_{2}$ and inhibit ALDH1A1 and ALDH3A1. 
Compound 29 has a large 2-oxo-2-phenylethoxy group at $\mathrm{R}_{2}$ and inhibits ALDH1A1, as well as demonstrating moderate inhibition of ALDH3A1.

Compound $\mathbf{3 0}$ has a terminal nitrile at $\mathrm{R}_{2}$ and inhibits ALDH1A1, ALDH2, and ALDH3A1 at similar levels. Compounds $\mathbf{3 1}$ and $\mathbf{3 2}$ both appear to selectively inhibit ALDH1A1, though 31 only inhibits $50 \%$ at $10 \mu \mathrm{M}$ most likely due to the large $\mathrm{N}$-phenylacetamide group at $\mathrm{R}_{2}$. Compoudn 32 contains a diethylamine in the same position and inhibits ALDH1A1 85\% at $10 \mu \mathrm{M}$. The last analogs went beyond altering the methyl 2-methoxypropanoate group or adding to the ring structure (33-37). Three of these compounds showed selectivity towards either ALDH1A1 or ALDH2. Compounds 34, which added a methane sulfonyl at $\mathrm{R}_{2}$ and a benzyl group at $\mathrm{R}_{6}$, and 37 , which added isobutyramide at $\mathrm{R}_{3}$, selectively inhibited ALDH1A1 and 36, which replaced the methyl 2-methoxypropanoate group with a hydrogen at $\mathrm{R}_{2}$ and added a bromine at $\mathrm{R}_{3}$ and ketoxime at $\mathrm{R}_{6}$, selectively inhibited ALDH2. Of the 33 analogs tested, seven showed potential selectivity for ALDH2 based off activity at $10 \mu \mathrm{M}$ compound $(7,9,10,11,23,26$, and 36) and two compounds showed selectivity and potency towards ALDH1A1 (32 and 34). These nine compounds, as well as the strong ALDH1A1 and ALDH2 inhibitor, 15, were selected for further analysis.

Due to the hydrophobic nature of the substrate binding site within the free enzyme species of ALDH2, the $\log \mathrm{P}$ values for the 33 analogs in addition to the initial four hits were examined (Tables 3 and 4). No direct correlation between $\log P$ values and inhibition of ALDH1A1 or ALDH2 could be discerned.

\section{$\mathrm{EC}_{50}$ Determination of Selected Analogs}

$\mathrm{EC}_{50}$ values were measured in regards to ALDH1A1, ALDH2, and ALDH3A1 for the ten selected compounds. Three compounds were excluded from further study for one of two reasons (Table 5). Both 9 and 23 are selective for $\mathrm{ALDH} 2\left(\mathrm{IC}_{50}=0.15 \pm 0.01 \mu \mathrm{M}\right.$ for each) but only partially inhibit the enzyme. We sought complete inhibitors of the isoenzymes so these analogs were not pursued further. Compound $\mathbf{2 6}$ was selective for ALDH2 versus ALDH1A1; however the compound was not characterized further as it partially inhibited ALDH3A1 activity.

The seven remaining compounds did not inhibit ALDH3A1 and fully inhibited ALDH1A1 and/or ALDH2. For these compounds $\mathrm{EC}_{50}$ values for ALDH1B1, ALDH1A2, and ALDH1A3 were also calculated in addition to those for ALDH1A1, ALDH2, and ALDH3A1 (Table 6).

Compounds 7, 10, and $\mathbf{1 1}$ had $\mathrm{IC}_{50}$ values for the ALDH1/2 family of isoenzymes very similar to the psoralen derivatives found through the high-throughput screen and were more potent than daidzin. No improvement to the selectivity for any one particular isoenzyme of the ALDH1/2 family was seen from adding the cyclopentyl, cyclcohexyl, or propyl group to the psoralen backbone. These additional groups would fit between Trp177 and Met174 in ALDH2, both of which are conserved throughout the ALDH1/2 family (Figure 2E). Compound 7 most likely doesn't inhibit ALDH1A1 due to the lack of the longer alkyl chain at $\mathrm{R}_{6}$ to anchor itself in the larger pocket. Compound 15 showed a $\sim 10$-fold preference towards inhibiting ALDH1A1 $\left(\mathrm{IC}_{50}=0.13 \pm 0.01 \mu \mathrm{M}\right)$ versus the other four ALDH1A/2 
isoenzymes. Compound 32 was found to be selectively inhibit ALDH1A1 $\left(\mathrm{IC}_{50}=0.76 \pm 0.07\right.$ $\mu \mathrm{M})$ with only minor effects on ALDH1A2. Compound $\mathbf{3 4}$ showed a preference towards ALDH1A1 $\left(\mathrm{IC}_{50}=2.8 \pm 0.1 \mu \mathrm{M}\right)$ while also inhibiting ALDH1B1, ALDH1A2, and ALDH1 A3 activity. 34 had no measurable effect on the activity of ALDH2. 36 selectively inhibited ALDH2 $\left(\mathrm{IC}_{50}=4.6 \pm 0.5 \mu \mathrm{M}\right)$ while also exhibiting two-fold activation of ALDH1 $\mathrm{A} 3\left(\mathrm{AC}_{50}=35 \pm 3 \mu \mathrm{M}\right)$. The inhibition properties of $\mathbf{1 5}, \mathbf{3 2}, \mathbf{3 4}$, and $\mathbf{3 6}$ were further explored through crystallographic and kinetic studies.

\section{Characterization of Compound 15 Binding}

The structure of $\mathbf{1 5}$ bound to ALDH1A1 was solved to a resolution of $1.70 \AA$ (Table 2). The structure shows that 15 binds within the substrate binding site of ALDH1A1 (Figure 4A). However, the psoralen backbone of $\mathbf{1 5}$ has shifted relative to the position of $\mathbf{2}$ bound to ALDH2 and is bound between Tyr297, Gly458, His293, and Phe290. As a consequence of this shift, the piperidine ring of the longer linker structure of $\mathbf{1 5}$ is positioned near the catalytic cysteine. Unlike the alkyl chain of $\mathbf{2}$, the piperidine ring in this position does not approach the $\mathrm{NAD}(\mathrm{H})$ binding site (Figures $3 \mathrm{~B}$ and $4 \mathrm{~B}$ ). Covariation experiments were used to evaluate the mechanistic differences between the binding of $\mathbf{1 5}$ to ALDH1A1 and to ALDH2. 15 was found to exhibit uncompetitive inhibition $\left(K_{i}=170 \pm 13 \mathrm{nM}\right)$ towards varied $\mathrm{NAD}^{+}$for $\mathrm{ALDH} 1 \mathrm{~A} 1$, which is consistent both with the distance between the NAD ${ }^{+}$ and 15 binding sites in ALDH1A1 and is expected for compounds that displace aldehyde substrates for ordered Bi Bi systems (Figure 4C). Uncompetitive inhibition would suggest that 15 binds only to the enzyme-coenzyme complex and not to the free enzyme. However, the structural data of $\mathbf{1 5}$ bound to the free enzyme appears to contradict the kinetic data, but the concentration ranges utilized for the two experiments did not overlap. The concentration of $\mathbf{1 5}$ used in the crystallography experiment was 400 -fold times the highest concentration utilized in the kinetic experiment $(200 \mu \mathrm{M}$ vs $0.5 \mu \mathrm{M})$. Thus, 15 appears to also bind to the free enzyme, but under the conditions of the steady-state kinetic experiments, this complex formation is kinetically insignificant and yields an uncompetitive pattern. A similar pattern of uncompetitive inhibition towards varied coenzyme in a kinetic experiment, compared to a complex between the free enzyme and inhibitor, was also observed with daidzin and ALDH2. ${ }^{36,37}$ Interestingly, 15 exhibited competitive inhibition $\left(\mathrm{K}_{\mathrm{i}}=1.1 \pm 0.1 \mu \mathrm{M}\right)$ towards varied $\mathrm{NAD}^{+}$for $\mathrm{ALDH} 2$ (Figure 4D). This result is similar to the mechanism exhibited by $\mathbf{2}$, suggesting 15 retains a binding relationship similar to $\mathbf{2}$ bound to ALDH2. These data would infer that the psoralen substructure of $\mathbf{1 5}$ binds to ALDH2 with the 1-acylpiperidine extending past Cys302 and posed toward the $\mathrm{NAD}^{+}$site, consistent with its competitive mode of inhibition. However, for ALDH1A1 the presence of Gly458 and its larger substrate binding pocket promotes a shift in binding mode and mechanism of inhibition for $\mathbf{1 5}$. The residue corresponding to Gly458 in the other four ALDH1/2 isoenzymes is either an asparagine or aspartate (D457 in ALDH2) which precludes the binding mode observed in ALDH1A1.

\section{Characterization of Compound 34 Binding}

The structure of the coumarin derivative $\mathbf{3 4}$ bound to ALDH1A1 was solved to a resolution of $1.70 \AA$ (Table 2). 34 binds within the substrate binding site (Figure $5 \mathrm{~A}$ ) and the lactone 
carbonyl is oriented towards the catalytic cysteine though not close enough to form a hydrogen bond like $\mathbf{2}$ in ALDH2 (Figure 5B). Although the electron density for the benzyl group of $\mathbf{3 4}$ was diffuse, the movement of Trp178 was reminiscent of the interaction between CM037 and ALDH1A1 in the same region, which also resulted in more diffuse density in this region. ${ }^{49}$ The additional benzyl group prevents the compound from binding closer to the catalytic cysteine. $\mathbf{3 4}$ does not inhibit ALDH2 and the mode of binding provides insight into this selectivity. The methane sulfonyl group of $\mathbf{3 4}$ binds in the pocket formed by Gly458 in ALDH1A1. This additional space only exists in ALDH1A1. The corresponding aspartate/asparagine residues in the other ALDH1/2 isoenzymes would force the coumarin scaffold to adopt a position like $\mathbf{2}$ in ALDH2. However, unlike the flexible linker in $\mathbf{1 5}$, the large benzyl ring proximal to the lactone cannot adopt a conformation to productively bind within the $\mathrm{NAD}^{+}$-binding cleft and the benzyl ring of $\mathbf{3 4}$ would encounter more steric hindrance from as the conserved tryptophan (Trp177 in ALDH2) since the residues surrounding Trp177 are bulkier in ALDH2.

\section{Characterization of Compound 32 Binding}

The structure of compound $\mathbf{3 2}$ bound to ALDH1A1 was solved to a resolution of $2.05 \AA$, with the ligand modeled at full occupancy in each of the eight monomers (Table 2). Surprisingly, 32 binds in the coenzyme binding cleft of ALDH1A1 (Figure 6A) between Pro227 and Val250 in a position similar to the adenine ring of NAD ${ }^{+}$(Figure 6B). The fact that 32 bound in the coenzyme binding site ALDH1A1 and the fact the crystal formed in a unique space group (P1) raises the possibility that the binding site might be influenced by the crystal environment. However, there are structural reasons for the selectivity between ALDH2 and ALDH1A1 in this binding location which suggest that binding within the coenzyme site is not an artifact of this particular crystal form (Figure 7). There are three amino acid substitutions of residues that directly contact $\mathbf{3 2}$ between ALDH1A1 and ALDH2. In particular, Ile249 in ALDH2 is Val250 in ALDH1A1 and although it is the addition of a single methyl group, the substitution narrows this side of the site enough to impede binding of the diethylamino substituent in ALDH2. In addition, Val252 and Ala233 in ALDH2 versus Leu253 and Ser234 in ALDH1A1 may loosen the contacts between the enzyme and $\mathbf{3 2}$ on the side away from Ile249 sufficiently that the binding energetics cannot overcome the steric clash at 249 through small shifts toward this side of the binding cleft. Covariation experiments were completed to further characterize the interaction of $\mathbf{3 2}$ with ALDH1A1. If $\mathbf{3 2}$ was bound to the $\mathrm{NAD}^{+}$binding site as seen in the crystal structure the inhibition profile versus varied $\mathrm{NAD}^{+}$would be predicted to be competitive. Surprisingly, 32 was found to be noncompetitive versus varied $\mathrm{NAD}^{+}$for ALDH1A1 with $\mathrm{K}_{\mathrm{i}}=1.2 \pm 0.1 \mu \mathrm{M}$ and noncompetitive versus varied acetaldehyde for ALDH1A1 with $\mathrm{K}_{\mathrm{i}}=0.87 \pm 0.04 \mu \mathrm{M}$ (Figure 6C) Although noncompetitive inhibition versus $\mathrm{NAD}^{+}$is inconsistent with the binding of $\mathbf{3 2}$ to the $\mathrm{NAD}^{+}$site, it is also a different mode of inhibition from compounds determined to bind in the substrate binding site, $\mathbf{2}$ (competitive) and $\mathbf{1 5}$ (uncompetitive), which suggests $\mathbf{3 2}$ binds differently than these other analogs. A similar compound to $\mathbf{3 2}$ was also tested to support the kinetic data of $\mathbf{3 2 . 3 0}$ is a coumarin derivative which essentially replaces the diethylamine of $\mathbf{3 2}$ with a terminal cyano group. $\mathbf{3 0}$ inhibits both ALDH1A1 and ALDH2 as Ile249 in ALDH2 does not provide selectivity against the linear cyano group in this binding position. $\mathbf{3 0}$ was found to be noncompetitive versus varied $\mathrm{NAD}^{+}$for 
ALDH1A1 and be noncompetitive (mixed-type) versus varied NAD ${ }^{+}$for ALDH2 (Figure 6D) which is consistent with the inhibition mode of 32. The reason behind the discrepancy between the kinetic data and structural data remains unclear. There is a possibility that 32 could bind to a different location in the enzyme when $\mathrm{NAD}^{+}$is present, and when $\mathrm{NAD}^{+}$is absent (as in the case of the structure) the compound prefers to bind in the $\mathrm{NAD}^{+}$binding site. The possibility of multiple binding sites is supported by the compound exhibiting noncompetitive inhibition towards varied $\mathrm{NAD}^{+}$or varied substrate (Figure 6C). This additional site could be the substrate binding site where $\mathbf{3 4}$, another coumarin derivative, binds.

\section{Characterization of Compound 36 Binding to ALDH2}

Compound 36 was the lone ALDH2-selective compound (apart from 4) discovered in this study. Covariation experiments were performed to determine how $\mathbf{3 6}$ inhibits ALDH2 in part due to the diverse inhibition profile of the other compounds towards ALDH1A1. 36 was found to competitively inhibit the binding of $\mathrm{NAD}^{+}$to $\mathrm{ALDH}_{2}$ with a $\mathrm{K}_{\mathrm{i}}=2.4 \pm 0.1 \mu \mathrm{M}$ (Figure 8). This result suggests that $\mathbf{3 6}$ also alters the conformation of the catalytic cysteine interfering with the productive binding of $\mathrm{NAD}^{+}$for catalysis similar to $\mathbf{2}$ and $\mathbf{3}$. If $\mathbf{3 6}$ were to adopt a similar conformation to that of $\mathbf{2}$, the ketoxime would occupy the same pocket as the propyl chain. The bromine would be oriented towards Met174 which could lead to a favorable interaction between the halogen substituent and the sulfur side chain atom. This potential interaction is not possible for the three ALDH1A isoenzymes since a glycine occupies the equivalent position. However, it is unclear what effect the equivalent glutamate in ALDH1B1 would have on 36 binding. The glutamate of ALDH1B1 could provide additional contacts for $\mathbf{3 6}$ binding, thus the rationale for selectivity towards ALDH2 by $\mathbf{3 6}$ is still unclear.

\section{Aromatic Binding Regions of ALDH1A1 and ALDH2}

Since the binding modes and kinetic data are distinct for particular compound/enzyme pairs, we reasoned that aspects of the substrate binding sites and differences in kinetic mechanism underlie these differences. In particular, structural comparisons of the three compoundenzyme complexes with compound in the substrate binding site highlighted the presence of two distinct aromatic binding "boxes" or "slots" (Figure 9). In ALDH2 the aromatic binding region is located between four phenylalanine residues $(170,296,459,465)$ near the catalytic cysteine and surround 2 . The isoflavone of the ALDH2 inhibitor daidzin binds in the same aromatic region as does the activator Alda-1. ${ }^{37,50}$ The aromatic binding region of

ALDH1A1 is wider, extends farther from the catalytic cysteine and is more surface exposed. Of the four phenylalanine residues near the catalytic cysteine in ALDH2, Phe459 is replaced by a valine and Phe 296 is replaced by a tyrosine. The aromatic binding region of ALDH1A1 has additional aromatic residues at His293 and Phe290. The corresponding residues in ALDH2 are Phe292 and Gln289. Although Phe292 in ALDH2 is aromatic, the side chain of Asp457 blocks any connection of the two binding sites, which makes the binding region in ALDH2 smaller than that in ALDH1A1, though the two regions do overlap. Compounds have the ability to bind throughout the aromatic region in ALDH1A1 due to Gly458, as previously described for the ALDH1A1 inhibitors CM037 and CM026. ${ }^{49}$ 
A second aromatic binding pocket exists in ALDH isoenzymes, namely the adenine binding cleft within the coenzyme binding site, where the adenine ring is positioned between a conserved proline (Pro227 in ALDH1A1) and beta-branched amino acid (Val250 in ALDH1A1). However, there is only one aromatic amino acid within this structural element (Phe244) with the remainder being primarily aliphatic side chains. Consequently, while the site is hydrophobic, there are fewer aromatic groups present in this region than in the substrate binding site which may reflect the more hydrophilic nature of the adenosine group of $\mathrm{NAD}^{+}$and lead to greater partitioning into the substrate site for most of these compounds. However, the structure of $\mathbf{3 2}$ shows that at least one of the compounds presented in this work can bind to the adenine site. Although, the kinetic data support binding at an additional site, most likely the aromatic binding region in the substrate binding site.

\section{CONCLUSION}

Selective compounds for the individual members of the ALDH1/2 family of isoenzymes would be useful in determining their relative contributions to biological function. This study characterized a series of aromatic lactones which inhibit the ALDH1/2 family with the goal of developing an ALDH2-selective inhibitor. The agreement between the structure activity relationships and structural studies show the interactions are both specific and selective. The psoralen and coumarin analogs take advantage of multiple aromatic binding regions of the ALDH1/2 isoenzymes. Although psoralen and coumarin derivatives can form Michael additions, all but two compounds tested have at least a methyl group at position $\mathrm{R}_{5}$ preventing such a reaction and thus eliminating the possibility of covalent inhibition by this means. Additionally, the kinetic and structural data demonstrate a non-covalent means of inhibition. Aromatics can bind within the hydrophobic substrate binding site as well as the $\mathrm{NAD}^{+}$binding site. The size of the compound and the neighboring residues help determine to which of the sites the compounds bind.

The differing binding modes of the derivatives make it somewhat challenging to predict where compounds will bind though some basic observations can be applied. The small coumarin analogs can bind in the $\mathrm{NAD}^{+}$binding site like 32, but once they reach a size threshold the compounds prefer the substrate binding site (e.g. 34). The absence of an amino acid side chain at Gly458 in ALDH1A1 allows for a larger aromatic binding area, best exemplified by $\mathbf{1 5}$. The unique aromatic binding regions linked to topological differences allow the same coumarin ring structure to be selective for different isoenzymes as substituents are varied to better match the target enzyme's structural features.

Surprisingly, 32 achieves selectivity towards ALDH1A1 by binding in the more highly conserved adenine binding site. Our initial screen was designed to avoid compounds which bound in the $\mathrm{NAD}^{+}$binding site as we reasoned these would have lower selectivity. 32 shows that selectivity is possible through binding in this location. $\mathbf{3 6}$ achieves selectivity towards ALDH2 while competitively inhibiting the productive binding of NAD ${ }^{+}$. Although the exact binding location of $\mathbf{3 6}$ is unknown, the compound most likely binds in similar position to 2 in the substrate binding pocket. 36 inhibits $\mathrm{ALDH} 2$ with similar $\mathrm{IC}_{50}$ values to daidzin in vitro and is more selective. Daidzin, previously described as an ALDH2-seletive inhibitor, inhibited the activity of three of the five ALDH1/2 isoenzymes. Several additional 
derivatives in this study improve upon daidzin's potency towards the ALDH1/2 isoenzymes in our assays. Future experiments will consist of further developing structure-activity relationships for $\mathbf{3 2}$ and $\mathbf{3 6}$ and determining their effects in various cellular models.

\section{EXPERIMENTAL SECTION}

\section{Materials}

All materials were purchased from Sigma-Aldrich (St. Louis, MO), unless otherwise noted. Compounds purchased from ChemDiv Corporation (San Diego, CA) and ChemBridge Corporation (San Diego, CA) were $>95 \%$ pure based on the spectra (either NMR or LC/MS) provided by the vendor. Following receipt from the vendor, their chemical identities were further verified by LC/MS to check for correct molecular weight in the Department of Chemistry, Indiana University-Purdue University, Indianapolis, IN or the Chemical Genomics Core Facility, Indiana University School of Medicine, Indianapolis, IN and used without further purification. Compound 22, purchased from Sigma-Aldrich, was not checked for purity by the company and daidzin, purchased from TZS Scientific LLC (Framingham, MA), was $>99 \%$ pure by their analysis, though with no accompanying spectra. For both of these compounds, the purity was checked by LC/MS and NMR in the Chemical Genomics Core Facility, Indiana University School of Medicine, Indianapolis, IN.

\section{Expression of ALDH isoenzymes}

Human ALDH1A1, ALDH1A2, ALDH2, ALDH1B1, ALDH3A1, and rat ALDH1L1 were prepared and purified as previously described. ${ }^{25,39,51,52}$ Human ALDH1A3 was prepared by one of two protocols. The first was by using the previous described method which yielded very little protein for enzymatic study. ${ }^{52}$ The second was using a His-tagged construct for full length ALDH1A3 generously provided by Jaume Farres which was subcloned into the pET-30Xa/LIC expression plasmid. His-tagged ALDH1A3 was produced and purified as previously described for ALDH3A1, except only a single passage on a nickel-NTA column was used for purification. ${ }^{39}$ The two ALDH1A3 constructs behaved similarly when used in kinetic experiments.

\section{Compound Inhibition and $\mathrm{IC}_{50}$ Determination}

Inhibition of ALDH isoenzymes by the compounds and the respective $\mathrm{IC}_{50}$ curves were determined by measuring the formation of $\mathrm{NAD}(\mathrm{P}) \mathrm{H}$ spectrophotometrically at $340 \mathrm{~nm}$ (molar extinction coefficient of $6220 \mathrm{M}^{-1} \mathrm{~cm}^{-1}$ ) on a Beckman DU-640 spectrophotometer using purified recombinant enzyme. All assays were performed at $25^{\circ} \mathrm{C}$ and were initiated by addition of the substrate after a 2 min incubation period. The assay components for these selectivity assays were designed to provide the maximal stringency toward ALDH2 such that the substrate concentration utilized was $>500$-fold above $\mathrm{K}_{\mathrm{M}}$ for ALDH2 while keeping below 15 -fold over $\mathrm{K}_{\mathrm{M}}$ for the other isoenzymes. ${ }^{43}$ For the ALDH1A family members and ALDH2, the assay included 100-200 $\mathrm{nM}$ enzyme, $200 \mu \mathrm{M} \mathrm{NAD}^{+}, 100 \mu \mathrm{M}$ propionaldehyde $\left(\mathrm{K}_{\mathrm{M}}\right.$ values $\sim 100 \mathrm{nM}$ for $\mathrm{ALDH} 2^{41}, \sim 50 \mu \mathrm{M}$ for ALDH1A1 ${ }^{53}$, ALDH1 $2^{54}$, and $\mathrm{K}_{\mathrm{M}}$ value for ALDH1 A3 determined empirically by varying propionaldehyde concentration ${ }^{41}$ ), and $1 \%$ DMSO in $25 \mathrm{mM}$ BES buffer, pH 7.5. The assay for ALDH1B1 used $500 \mu \mathrm{M} \mathrm{NAD}{ }^{+}$and $200 \mu \mathrm{M}$ propionaldehyde $\left(\mathrm{K}_{\mathrm{M}} \sim 14 \mu \mathrm{M}\right)^{55}$. For ALDH4A1 the assay included $500 \mu \mathrm{M}$ 
$\mathrm{NAD}^{+}$and $20 \mathrm{mM}$ propionaldehyde $\left(\mathrm{K}_{\mathrm{M}} \sim 9 \mathrm{mM}\right)^{56}$. For ALDH5A1 the assay included 200 $\mu \mathrm{M} \mathrm{NAD}^{+}$and $2 \mathrm{mM}$ propionaldehyde $\left(\mathrm{K}_{\mathrm{M}} \sim 600 \mu \mathrm{M}\right)^{57}$. For ALDH1L1 the assay included $500 \mu \mathrm{M} \mathrm{NADP}^{+}$and $4 \mathrm{mM}$ propionaldehyde $\left(\mathrm{K}_{\mathrm{M}} \sim 700 \mu \mathrm{M}\right)^{58}$. For ALDH3A1, the assay included the commonly utilized substrate benzaldehyde at $300 \mu \mathrm{M}\left(\mathrm{K}_{\mathrm{M}} \sim 200 \mu \mathrm{M}\right)^{59}$ alongside $300 \mu \mathrm{M} \mathrm{NADP}{ }^{+}, 20 \mathrm{nM}$ ALDH3A1 and 1\% DMSO. Assays for ALDH3A1, ALDH4A1, and ALDH5A1 were performed in $100 \mathrm{mM}$ sodium phosphate buffer, $\mathrm{pH}$ 7.5. All compounds were soluble in assays up to $100 \mu \mathrm{M}$ and did not interfere with the analytical output measured by the assays unless otherwise noted. Data were fit to the four parameter $\mathrm{EC}_{50}$ equation using SigmaPlot (v12) and the values represent the mean/SEM of three independent experiments (each $n=3$ ).

\section{Selection and Characterization of Analogs of Initial Inhibitors}

Thirty-three additional psoralen and coumarin derivatives were ordered from ChemDiv, ChemBridge, and Sigma-Aldrich to build structure-activity relationships. Compounds were initially tested for their effect on the oxidation of aldehyde substrate by ALDH1A1, ALDH2, and ALDH3A1. Compounds which showed potential selectivity for ALDH1A1 or $\mathrm{ALDH} 2$ were examined further by measuring $\mathrm{EC}_{50}$ values for each of the three enzymes. $\mathrm{EC}_{50}$ curves for ALDH1A2, ALDH1A3, and ALDH1B1 inhibition were determined for compounds which continued to show selectivity towards ALDH2 or ALDH1A1. Data were fit to the four parameter $\mathrm{EC}_{50}$ equation using SigmaPlot (v12) and the values represent the mean/SEM of three independent experiments.

\section{Steady-State Kinetic Characterization with ALDH1A1 and ALDH2}

The mode of inhibition towards varied coenzyme $\left(\mathrm{NAD}^{+}\right)$was determined via steady-state kinetics by varying inhibitor and coenzyme concentrations at fixed substrate concentrations. Dehydrogenase activity was measured spectrophotometrically by measuring the formation of NADH at $340 \mathrm{~nm}$ (molar extinction coefficient of $6220 \mathrm{M}^{-1} \mathrm{~cm}^{-1}$ ) on a Beckman DU-640 spectrophotomer. All assays included $100-200 \mathrm{nM}$ enzyme, $1 \mathrm{mM}$ propionaldehyde, and $1 \%$ DMSO in $25 \mathrm{mM}$ BES buffer, $\mathrm{pH} 7.5$ at $25^{\circ} \mathrm{C}$. For ALDH2 ranges of $15-400 \mu \mathrm{M} \mathrm{NAD}{ }^{+}$and either $0-100 \mathrm{nM} \mathrm{2,0} \mathrm{-} 1 \mu \mathrm{M} \mathrm{3}, 0-2 \mu \mathrm{M} \mathrm{4}, 0-10 \mu \mathrm{M} \mathrm{15}, 0-$ $50 \mu \mathrm{M} \mathrm{30}$, or $0-10 \mu \mathrm{M} 36$ were used. For ALDH1A1 ranges of $20-200 \mu \mathrm{M} \mathrm{NAD}^{+}$and either $0-500 \mathrm{nM} \mathrm{15}, 0-50 \mu \mathrm{M} \mathrm{30}$, or $0-4 \mu \mathrm{M} 32$ were used. The mode of inhibition of 32 toward varied acetaldehyde was determined via steady-state kinetics by varying inhibitor and substrate concentrations at fixed coenzyme concentrations. Assays included $300 \mathrm{nM}$ ALDH1A1, $1 \mathrm{mM} \mathrm{NAD}+, 30-500 \mu \mathrm{M}$ acetaldehyde, $0-1.5 \mu \mathrm{M} \mathrm{32}$, and $1 \%$ DMSO in 25 $\mathrm{mM}$ BES buffer, $\mathrm{pH} 7.5$ at $25^{\circ} \mathrm{C}$. All data were fit to the tight binding or single substratesingle inhibitor non-linear velocity expressions for competitive, non-competitive, mixed type non-competitive, and uncompetitive inhibition using SigmaPlot (v12, Enzyme Kinetics Module) to evaluate goodness of fit. Lineweaver-Burk plots were generated using SigmaPlot(v12) to better visualize the inhibition. All data represent the mean/SEM of at least three independent experiments (each $n=3$ ). 


\title{
Crystallization of ALDH1A1 and ALDH2 in Complex with Compounds
}

Crystals of ALDH2 in complex with 2 were grown by equilibrating $8 \mathrm{mg} / \mathrm{mL}$ ALDH2 and $200 \mu \mathrm{M} 2$ in $2 \%$ DMSO with $100 \mathrm{mM}$ Na-ACES, pH 6.4, $100 \mathrm{mM}$ guanidine-HCl, $10 \mathrm{mM}$ $\mathrm{MgCl}_{2}, 4 \mathrm{mM}$ dithiothreitol, and 18\% PEG 6000. Crystals of ALDH1A1 were grown by equilibrating $3 \mathrm{mg} / \mathrm{mL}$ ALDH1A1 with 100mM Na-BisTris, pH 6.2 - 6.6, $9-11 \%$ PEG3350, $200 \mathrm{mM} \mathrm{NaCl}$, and $5 \mathrm{mM} \mathrm{YbCl}_{3}$. Crystals of ALDH1A1 in complex with 15 and 32 were grown in the presence of $200 \mu \mathrm{M}$ compound and $2 \%$ (v/v) DMSO (cocrystallization). The crystal of ALDH1A1 in complex with $\mathbf{3 4}$ was prepared by soaking an apo-ALDH1A1 crystal overnight with crystallization solution containing $500 \mu \mathrm{M} 34$ with $2 \%(\mathrm{v} / \mathrm{v})$ DMSO. Crystals were cryoprotected for flash-freezing with $18 \%(\mathrm{v} / \mathrm{v})$ ethylene glycol for ALDH2 and 20\% (v/v) ethylene glycol for ALDH1A1. Diffraction data were collected at Beamline 19-ID operated by the Structural Biology Consortium at the Advanced Proton Source (APS), Argonne National Laboratory. Diffraction data were indexed, integrated, and scaled using HKL2000 or HKL3000 program suites. ${ }^{60}$ HKL2000 version 0.96 was used for the structure of ALDH2 in complex with 2, HKL2000 version 706 was used for the structures of ALDH1A1 in complex with $\mathbf{1 5}$ and 34, and HKL3000 version 712 was used for the structure of ALDH1A1 in complex with 32. The CCP4 program suite was used for molecular replacement and refinement with the human apo-ALDH2 structure (PDB Code 3N80) as a model for ALDH2 and human apo-ALDH1A1 structure (PDB Code 4WJ9) for ALDH1A1 ${ }^{61}$ The Coot molecular graphic application was used for model building. ${ }^{62}$ An oxidized cysteine residue (CSO) was modeled in the active site at position 303 for the structures of ALDH1A1 in complex with 15 and 34. The TLSMD (Translation/Libration/ Screw Motion Determination) server was used to determine dynamic properties of ALDH1A1.63, 64

\section{Supplementary Material}

Refer to Web version on PubMed Central for supplementary material.

\section{Acknowledgments}

The authors would like to thank Dr. Cindy Morgan, Dr. Bibek Parajuli, Colin Tully, Mikail Chtcherbinine, and Lanmin Zhai for help with the production and purification of the various ALDH isoenzymes, Dr. Jaume Farres for providing the His-tagged ALDH1A3 expression clone, Dr. Sergey Krupenko for providing the rat ALDH1L1 expression clone, Drs. Daria Mochly-Rosen and Che-Hong Chen for providing the expression clones for ALDH4A1 and ALDH5A1, and Drs. Karl Dria and Lifan Zeng for assistance with LC/MS. Results shown in this report are derived from work performed at Argonne National Laboratory, Structural Biology Center at the Advanced Photon Source. Argonne is operated by UChicago Argonne, LLC, for the U.S. Department of Energy, Office of Biological and Environmental Research under contract DE-AC02- 06CH11357. This research used resources of the Advanced Photon Source, a U.S. Department of Energy (DOE) Office of Science User Facility operated for the DOE Office of Science by Argonne National Laboratory under Contract No. DEAC02-06CH11357. This work was supported by the U.S. National Institutes of Health (grants RO1AA018123 and R21CA198409 to T.D.H.).

\section{ABBREVIATIONS USED}

\author{
ALDH aldehyde dehydrogenase \\ BES N,N-Bis(2-hydroxyethyl)-2-aminoethanesulfonic acid
}


ACES N-(2-acetamido)-2-aminoethanesulfonic acid

BisTris Bis(2-hydroxyethyl)aminotris(hydroxymethyl)methane

\section{References}

1. Cheng G, Shi Y, Sturla SJ, Jalas JR, McIntee EJ, Villalta PW, Wang M, Hecht SS. Reactions of formaldehyde plus acetaldehyde with deoxyguanosine and DNA: formation of cyclic deoxyguanosine adducts and formaldehyde cross-links. Chem Res Toxicol. 2003; 16:145-152. [PubMed: 12588185]

2. Brooks PJ, Zakhari S. Acetaldehyde and the genome: beyond nuclear DNA adducts and carcinogenesis. Environ Mol Mutagen. 2014; 55:77-91. [PubMed: 24282063]

3. O'Brien PJ, Siraki AG, Shangari N. Aldehyde sources, metabolism, molecular toxicity mechanisms, and possible effects on human health. Crit Rev Toxicol. 2005; 35:609-662. [PubMed: 16417045]

4. Vasiliou V, Pappa A, Estey T. Role of human aldehyde dehydrogenases in endobiotic and xenobiotic metabolism. Drug Metab Rev. 2004; 36:279-299. [PubMed: 15237855]

5. Marchitti SA, Brocker C, Stagos D, Vasiliou V. Non-P450 aldehyde oxidizing enzymes: the aldehyde dehydrogenase superfamily. Expert Opin Drug Metab Toxicol. 2008; 4:697-720. [PubMed: 18611112]

6. Kedishvili NY, Popov KM, Rougraff PM, Zhao Y, Crabb DW, Harris RA. CoA-dependent methylmalonate-semialdehyde dehydrogenase, a unique member of the aldehyde dehydrogenase superfamily. cDNA cloning, evolutionary relationships, and tissue distribution. J Biol Chem. 1992; 267:19724-19729. [PubMed: 1527093]

7. Vasiliou V, Bairoch A, Tipton KF, Nebert DW. Eukaryotic aldehyde dehydrogenase (ALDH) genes: human polymorphisms, and recommended nomenclature based on divergent evolution and chromosomal mapping. Pharmacogenetics. 1999; 9:421-434. [PubMed: 10780262]

8. Rizzo WB, Carney G. Sjogren-Larsson syndrome: diversity of mutations and polymorphisms in the fatty aldehyde dehydrogenase gene (ALDH3A2). Hum Mutat. 2005; 26:1-10. [PubMed: 15931689]

9. Geraghty MT, Vaughn D, Nicholson AJ, Lin WW, Jimenez-Sanchez G, Obie C, Flynn MP, Valle D, $\mathrm{Hu} \mathrm{CA}$. Mutations in the delta1-pyrroline 5-carboxylate dehydrogenase gene cause type II hyperprolinemia. Hum Mol Genet. 1998; 7:1411-1415. [PubMed: 9700195]

10. Pearl PL, Gibson KM, Cortez MA, Wu Y, Carter Snead O 3rd, Knerr I, Forester K, Pettiford JM, Jakobs C, Theodore WH. Succinic semialdehyde dehydrogenase deficiency: lessons from mice and men. J Inherited Metab Dis. 2009; 32:343-352. [PubMed: 19172412]

11. Stockler S, Plecko B, Gospe SM Jr, Coulter-Mackie M, Connolly M, van Karnebeek C, MercimekMahmutoglu S, Hartmann H, Scharer G, Struijs E, Tein I, Jakobs C, Clayton P, Van Hove JL. Pyridoxine dependent epilepsy and antiquitin deficiency: clinical and molecular characteristics and recommendations for diagnosis, treatment and follow-up. Mol Genet Metab. 2011; 104:48-60. [PubMed: 21704546]

12. Duester G. Families of retinoid dehydrogenases regulating vitamin A function: production of visual pigment and retinoic acid. Eur J Biochem. 2000; 267:4315-4324. [PubMed: 10880953]

13. Hilton J. Role of aldehyde dehydrogenase in cyclophosphamide-resistant L1210 leukemia. Cancer Res. 1984; 44:5156-5160. [PubMed: 6488175]

14. Moreb JS, Mohuczy D, Ostmark B, Zucali JR. RNAi-mediated knockdown of aldehyde dehydrogenase class-1A 1 and class-3A1 is specific and reveals that each contributes equally to the resistance against 4-hydroperoxycyclophosphamide. Cancer Chemother Pharmacol. 2007; 59:127136. [PubMed: 16614850]

15. Condello S, Morgan CA, Nagdas S, Cao L, Turek J, Hurley TD, Matei D. $\beta$-Catenin-regulated ALDH1A1 is a target in ovarian cancer spheroids. Oncogene. 2015; 34:2297-2308. [PubMed: 24954508]

16. Niederreither K, Subbarayan V, Dolle P, Chambon P. Embryonic retinoic acid synthesis is essential for early mouse post-implantation development. Nat Genet. 1999; 21:444-448. [PubMed: 10192400] 
17. Dupe V, Matt N, Garnier JM, Chambon P, Mark M, Ghyselinck NB. A newborn lethal defect due to inactivation of retinaldehyde dehydrogenase type 3 is prevented by maternal retinoic acid treatment. Proc Natl Acad Sci U S A. 2003; 100:14036-14041. [PubMed: 14623956]

18. Klyosov AA, Rashkovetsky LG, Tahir MK, Keung WM. Possible role of liver cytosolic and mitochondrial aldehyde dehydrogenases in acetaldehyde metabolism. Biochemistry. 1996; 35:4445-4456. [PubMed: 8605194]

19. Ueshima Y, Matsuda Y, Tsutsumi M, Takada A. Role of the aldehyde dehydrogenase-1 isozyme in the metabolism of acetaldehyde. Alcohol Alcohol Suppl. 1993; 1B:15-19. [PubMed: 8003124]

20. Stewart MJ, Malek K, Xiao Q, Dipple KM, Crabb DW. The novel aldehyde dehydrogenase gene, ALDH5, encodes an active aldehyde dehydrogenase enzyme. Biochem Biophys Res Commun. 1995; 211:144-151. [PubMed: 7779080]

21. Farres J, Wang X, Takahashi K, Cunningham SJ, Wang TT, Weiner H. Effects of changing glutamate 487 to lysine in rat and human liver mitochondrial aldehyde dehydrogenase. A model to study human (Oriental type) class 2 aldehyde dehydrogenase. J Biol Chem. 1994; 269:1385413860. [PubMed: 7910607]

22. Singh S, Arcaroli J, Chen Y, Thompson DC, Messersmith W, Jimeno A, Vasiliou V. ALDH1B1 is crucial for colon tumorigenesis by modulating Wnt/beta-catenin, Notch and PI3K/Akt signaling pathways. PLoS One. 2015; 10:e0121648. [PubMed: 25950950]

23. Anastasiou V, Ninou E, Alexopoulou D, Stertmann J, Muller A, Dahl A, Solimena M, Speier S, Serafimidis I, Gavalas A. Aldehyde dehydrogenase activity is necessary for beta cell development and functionality in mice. Diabetologia. 2016; 59:139-150. [PubMed: 26518685]

24. Marchitti SA, Deitrich RA, Vasiliou V. Neurotoxicity and metabolism of the catecholamine-derived 3,4-dihydroxyphenylacetaldehyde and 3,4-dihydroxyphenylglycolaldehyde: the role of aldehyde dehydrogenase. Pharmacol Rev. 2007; 59:125-150. [PubMed: 17379813]

25. Hammen PK, Allali-Hassani A, Hallenga K, Hurley TD, Weiner H. Multiple conformations of NAD and NADH when bound to human cytosolic and mitochondrial aldehyde dehydrogenase. Biochemistry. 2002; 41:7156-7168. [PubMed: 12033950]

26. Farres J, Wang TT, Cunningham SJ, Weiner H. Investigation of the active site cysteine residue of rat liver mitochondrial aldehyde dehydrogenase by site-directed mutagenesis. Biochemistry. 1995; 34:2592-2598. [PubMed: 7873540]

27. Wang X, Weiner H. Involvement of glutamate 268 in the active site of human liver mitochondrial (class 2) aldehyde dehydrogenase as probed by site-directed mutagenesis. Biochemistry. 1995; 34:237-243. [PubMed: 7819202]

28. Eng MY, Luczak SE, Wall TL. ALDH2, ADH1B, and ADH1C genotypes in Asians: a literature review. Alcohol Res Health. 2007; 30:22-27. [PubMed: 17718397]

29. Harada S, Agarwal DP, Goedde HW. Aldehyde dehydrogenase deficiency as cause of facial flushing reaction to alcohol in Japanese. Lancet. 1981; 2:982.

30. Peng GS, Wang MF, Chen CY, Luu SU, Chou HC, Li TK, Yin SJ. Involvement of acetaldehyde for full protection against alcoholism by homozygosity of the variant allele of mitochondrial aldehyde dehydrogenase gene in Asians. Pharmacogenetics. 1999; 9:463-476. [PubMed: 10780266]

31. Koppaka V, Thompson DC, Chen Y, Ellermann M, Nicolaou KC, Juvonen RO, Petersen D, Deitrich RA, Hurley TD, Vasiliou V. Aldehyde dehydrogenase inhibitors: a comprehensive review of the pharmacology, mechanism of action, substrate specificity, and clinical application. Pharmacol Rev. 2012; 64:520-539. [PubMed: 22544865]

32. Lipsky JJ, Shen ML, Naylor S. In vivo inhibition of aldehyde dehydrogenase by disulfiram. Chem Biol Interact. 2001; 130-132:93-102.

33. Shen ML, Johnson KL, Mays DC, Lipsky JJ, Naylor S. Determination of in vivo adducts of disulfiram with mitochondrial aldehyde dehydrogenase. Biochem Pharmacol. 2001; 61:537-545. [PubMed: 11239496]

34. Goldstein M, Anagnoste B, Lauber E, McKeregham MR. Inhibition of dopamine- $\beta$-hydroxylase by disulfiram. Life Sci. 1964; 3:763-767.

35. Chen D, Cui QC, Yang H, Dou QP. Disulfiram, a clinically used anti-alcoholism drug and copperbinding agent, induces apoptotic cell death in breast cancer cultures and xenografts via inhibition of the proteasome activity. Cancer Res. 2006; 66:10425-10433. [PubMed: 17079463] 
36. Keung WM, Vallee BL. Daidzin: a potent, selective inhibitor of human mitochondrial aldehyde dehydrogenase. Proc Natl Acad Sci U S A. 1993; 90:1247-1251. [PubMed: 8433985]

37. Lowe ED, Gao GY, Johnson LN, Keung WM. Structure of daidzin, a naturally occurring antialcohol-addiction agent, in complex with human mitochondrial aldehyde dehydrogenase. J Med Chem. 2008; 51:4482-4487. [PubMed: 18613661]

38. Khanna M, Chen CH, Kimble-Hill A, Parajuli B, Perez-Miller S, Baskaran S, Kim J, Dria K, Vasiliou V, Mochly-Rosen D, Hurley TD. Discovery of a novel class of covalent inhibitor for aldehyde dehydrogenases. J Biol Chem. 2011; 286:43486-43494. [PubMed: 22021038]

39. Morgan CA, Parajuli B, Buchman CD, Dria K, Hurley TD. N,N-diethylaminobenzaldehyde (DEAB) as a substrate and mechanism-based inhibitor for human ALDH isoenzymes. Chem -Biol Interact. 2015; 234:18-28. [PubMed: 25512087]

40. Luo M, Gates KS, Henzl MT, Tanner JJ. Diethylaminobenzaldehyde is a covalent, irreversible inactivator of ALDH7A1. ACS Chem Biol. 2015; 10:693-697. [PubMed: 25554827]

41. Buchman CD, Mahalingan KK, Hurley TD. Discovery of a series of aromatic lactones as ALDH1/2-directed inhibitors. Chem -Biol Interact. 2015; 234:38-44. [PubMed: 25641190]

42. Arolfo MP, Overstreet DH, Yao L, Fan P, Lawrence AJ, Tao G, Keung WM, Vallee BL, Olive MF, Gass JT, Rubin E, Anni H, Hodge CW, Besheer J, Zablocki J, Leung K, Blackburn BK, Lange LG, Diamond I. Suppression of heavy drinking and alcohol seeking by a selective ALDH-2 inhibitor. Alcohol: Clin Exp Res. 2009; 33:1935-1944. [PubMed: 19673742]

43. Klyosov AA. Kinetics and specificity of human liver aldehyde dehydrogenases toward aliphatic, aromatic, and fused polycyclic aldehydes. Biochemistry. 1996; 35:4457-4467. [PubMed: 8605195]

44. Morgan CA, Hurley TD. Development of a high-throughput in vitro assay to identify selective inhibitors for human ALDH1A1. Chem -Biol Interact. 2015; 234:29-37. [PubMed: 25450233]

45. MacGibbon AK, Blackwell LF, Buckley PD. Kinetics of sheep-liver cytoplasmic aldehyde dehydrogenase. Eur J Biochem. 1977; 77:93-100. [PubMed: 20307]

46. MacGibbon AK, Blackwell LF, Buckley PD. Pre-steady-state kinetic studies on cytoplasmic sheep liver aldehyde dehydrogenase. Biochem J. 1977; 167:469-477. [PubMed: 23112]

47. D’Ambrosio K, Pailot A, Talfournier F, Didierjean C, Benedetti E, Aubry A, Branlant G, Corbier C. The first crystal structure of a thioacylenzyme intermediate in the ALDH family: new coenzyme conformation and relevance to catalysis. Biochemistry. 2006; 45:2978-2986. [PubMed: 16503652]

48. Perez-Miller SJ, Hurley TD. Coenzyme isomerization is integral to catalysis in aldehyde dehydrogenase. Biochemistry. 2003; 42:7100-7109. [PubMed: 12795606]

49. Morgan CA, Hurley TD. Characterization of two distinct structural classes of selective aldehyde dehydrogenase 1A1 inhibitors. J Med Chem. 2015; 58:1964-1975. [PubMed: 25634381]

50. Perez-Miller S, Younus H, Vanam R, Chen CH, Mochly-Rosen D, Hurley TD. Alda-1 is an agonist and chemical chaperone for the common human aldehyde dehydrogenase 2 variant. Nat Struct Mol Biol. 2010; 17:159-164. [PubMed: 20062057]

51. Parajuli B, Kimble-Hill AC, Khanna M, Ivanova Y, Meroueh S, Hurley TD. Discovery of novel regulators of aldehyde dehydrogenase isoenzymes. Chem -Biol Interact. 2011; 191:153-158. [PubMed: 21349255]

52. Parajuli B, Georgiadis TM, Fishel ML, Hurley TD. Development of selective inhibitors for human aldehyde dehydrogenase 3A1 (ALDH3A1) for the enhancement of cyclophosphamide cytotoxicity. ChemBioChem. 2014; 15:701-712. [PubMed: 24677340]

53. Xiao T, Zhang M, Ansari N. Studies on aldehyde dehydrogenase 1(ALDH1A1), a crucial enzyme in maintaining the lens clarity under oxidative stress. Invest Ophthalmol Visual Sci. 2005; 46:3851.

54. Wang X, Penzes P, Napoli JL. Cloning of a cDNA encoding an aldehyde dehydrogenase and its expression in Escherichia coli. Recognition of retinal as substrate. J Biol Chem. 1996; 271:1628816293. [PubMed: 8663198]

55. Stagos D, Chen Y, Brocker C, Donald E, Jackson BC, Orlicky DJ, Thompson DC, Vasiliou V. Aldehyde dehydrogenase 1B1: molecular cloning and characterization of a novel mitochondrial 
acetaldehyde-metabolizing enzyme. Drug Metab Dispos. 2010; 38:1679-1687. [PubMed: 20616185]

56. Forte-McRobbie CM, Pietruszko R. Purification and characterization of human liver "high Km" aldehyde dehydrogenase and its identification as glutamic gamma-semialdehyde dehydrogenase. J Biol Chem. 1986; 261:2154-2163. [PubMed: 3944130]

57. Ryzlak MT, Pietruszko R. Human brain glyceraldehyde-3-phosphate dehydrogenase, succinic semialdehyde dehydrogenase and aldehyde dehydrogenase isozymes: substrate specificity and sensitivity to disulfiram. Alcohol: Clin Exp Res. 1989; 13:755-761. [PubMed: 2690658]

58. Krupenko SA, Wagner C, Cook RJ. Expression, purification, and properties of the aldehyde dehydrogenase homologous carboxyl-terminal domain of rat 10-formyltetrahydrofolate dehydrogenase. J Biol Chem. 1997; 272:10266-10272. [PubMed: 9092577]

59. Pappa A, Estey T, Manzer R, Brown D, Vasiliou V. Human aldehyde dehydrogenase 3A1 (ALDH3A1): biochemical characterization and immunohistochemical localization in the cornea. Biochem J. 2003; 376:615-623. [PubMed: 12943535]

60. Otwinowski, Z., Minor, W. Processing of X-ray diffraction data collected in oscillation mode. In: Abselon, J.Simon, M.Carer, C., Sweet, R., editors. Methods in Enyzmology: Macromolecular Crystallography, Part A. Vol. 276. Academic Press; Cambridge, MA: 1997. p. 307-326.

61. Bailey S. The Ccp4 Suite - Programs for Protein Crystallography. Acta Crystallogr, Sect D: Biol Crystallogr. 1994; 50:760-763. [PubMed: 15299374]

62. Emsley P, Cowtan K. Coot: model-building tools for molecular graphics. Acta Crystallogr, Sect D: Biol Crystallogr. 2004; 60:2126-2132. [PubMed: 15572765]

63. Painter J, Merritt EA. Optimal description of a protein structure in terms of multiple groups undergoing TLS motion. Acta Crystallogr, Sect D: Biol Crystallogr. 2006; 62:439-450. [PubMed: 16552146]

64. Painter J, Merritt EA. A molecular viewer for the analysis of TLS rigid-body motion in macromolecules. Acta Crystallogr Sect D: Biol Crystallogr. 2005; 61:465-471. [PubMed: 15809496] 


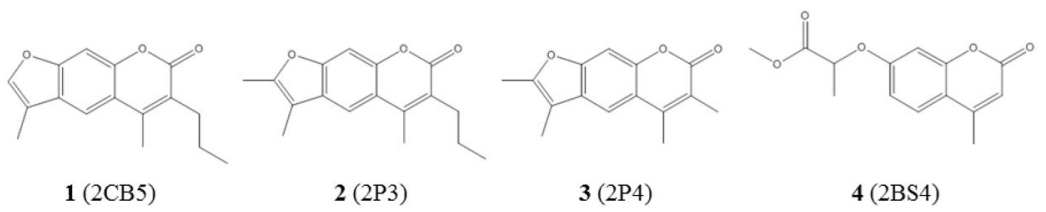

Figure 1.

Structures of the four partially characterized aromatic lactones. 

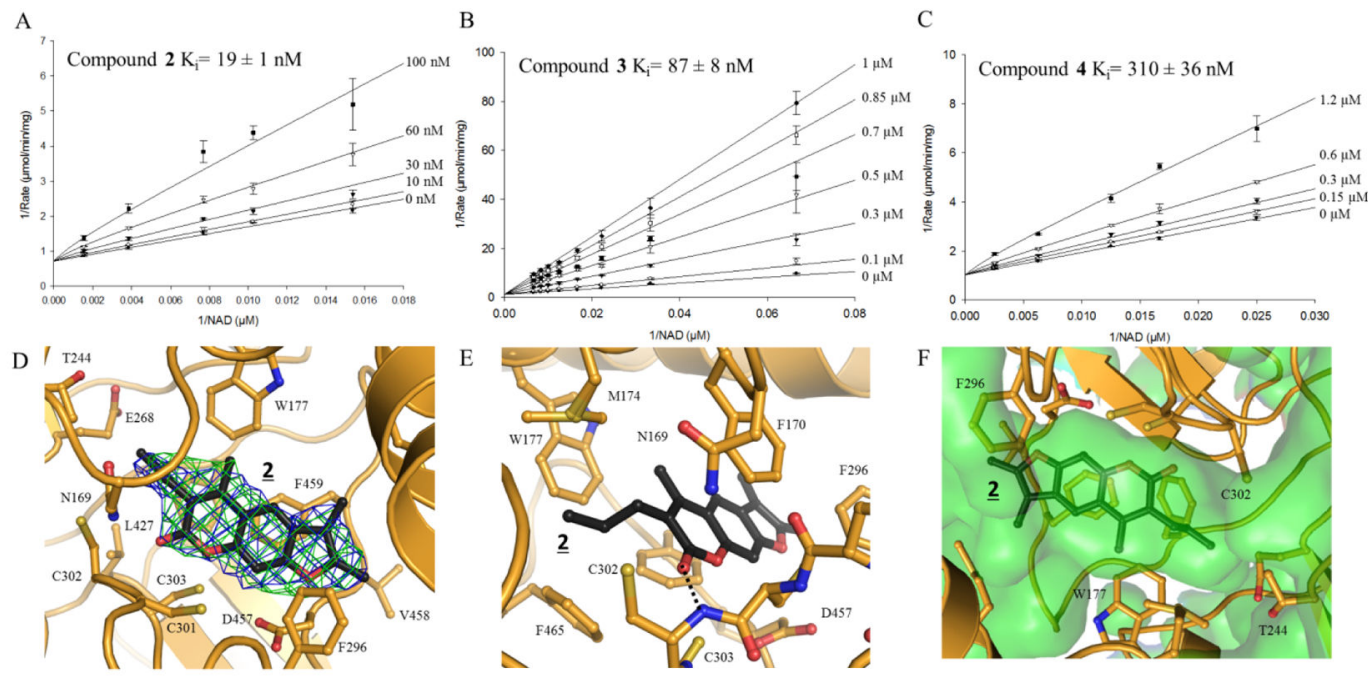

Figure 2. The binding characteristics of substituted psoralens to ALDH2 Lineweaver-Burk representations for the competitive inhibition patterns of compounds 2 (A), 3 (B), and 4 (C) versus varied $\mathrm{NAD}^{+}$at saturating concentrations of propionaldehyde (1 $\mathrm{mM})$. The reported $\mathrm{K}_{\mathrm{i}}$ values are the mean/SEM of at least three independent experiments( $\mathrm{n} 3$ ). (D) Representative electron density map for $\mathbf{2}$ bound to ALDH2 with the original figure-of-merit weighted $\mathrm{F}_{\mathrm{O}}-\mathrm{F}_{\mathrm{c}}$ map in green contoured at 2.5 standard deviations and the original figure-of-merit weighted $2 \mathrm{~F}_{\mathrm{o}}-\mathrm{F}_{\mathrm{c}}$ map in blue contoured at 1.0 standard deviations obtained after initial refinement before addition of solvent or ligand to the structure factor calculations. (E) Hydrogen bond between lactone carbonyl of 2 and peptide nitrogen of Cys302 shown as black dashed line. (F) 2 binds within the catalytic tunnel of ALDH2. The structure of ALDH2 is displayed using a ribbons and side chain representation with the molecular surface of the substrate binding site displayed in transparent green. The orientation of $\mathbf{2}$ in this panel is chosen to match the orientation of the compounds in Table 3 to better visualize the SAR. 


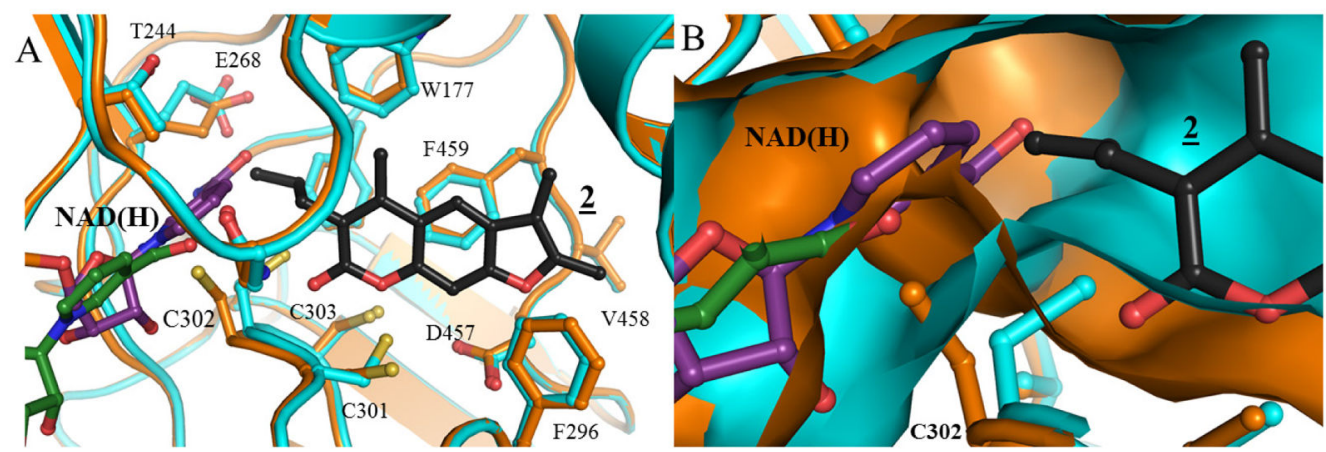

Figure 3. Potential interactions between psoralens and the active site of ALDH2

(A) Overlay of 2 (black) bound to ALDH2 (orange) and NAD $/ \mathrm{NADH}$ (purple/green) bound to ALDH2 (cyan). (B) Expanded view of the same overlay showing surface of ALDH2 for each binding partner using the same color scheme as in panel A. (PDB ID codes 1000, $1002,1004)$ 

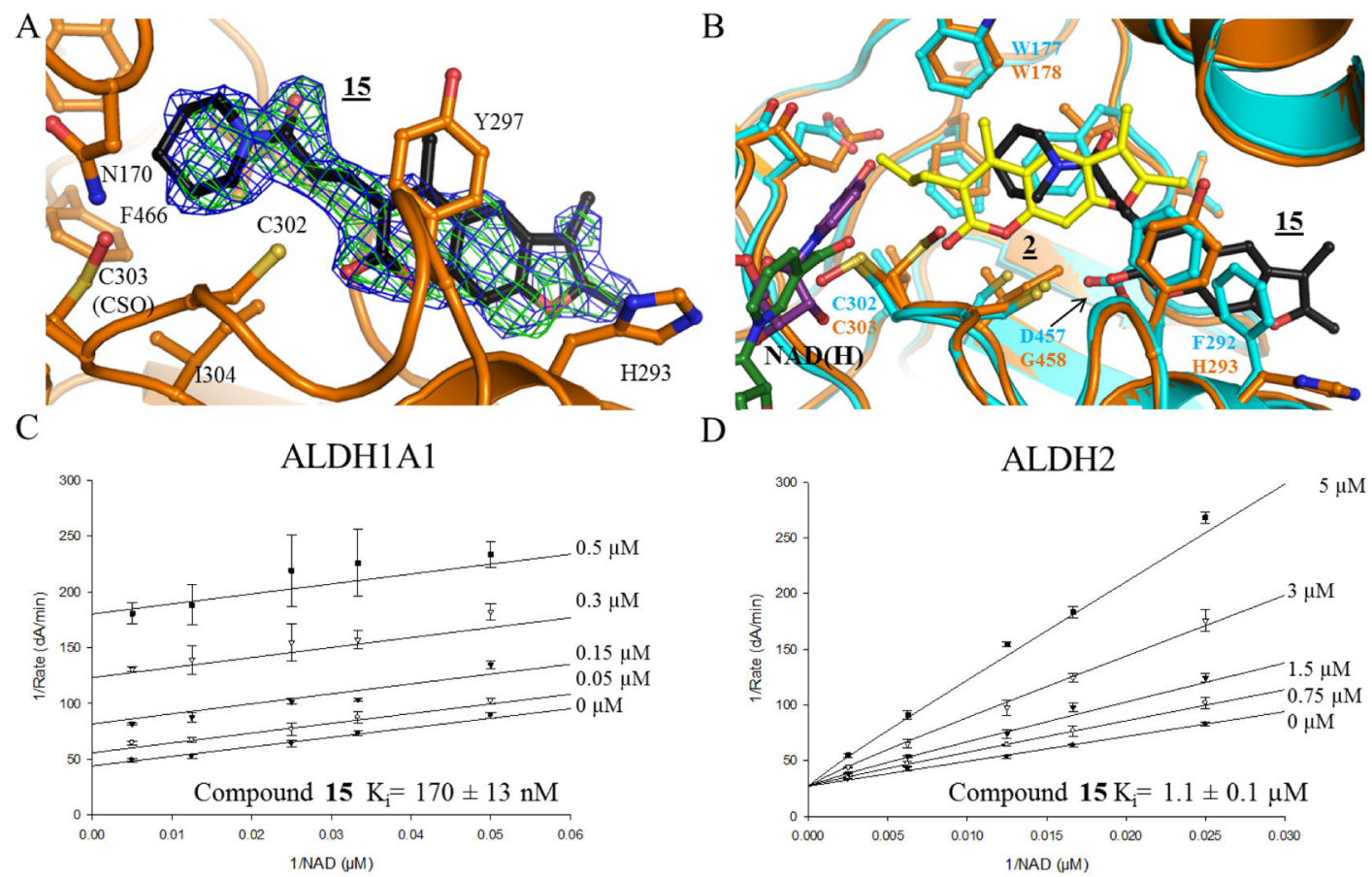

D

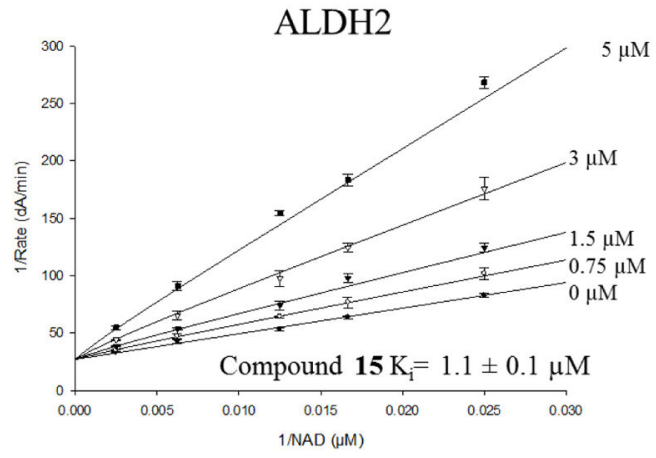

Figure 4. Binding characteristics of compound 15

(A) Electron density map of $\mathbf{1 5}$ bound to ALDH1A1 with the original figure-of-merit weighted $\mathrm{F}_{\mathrm{O}}-\mathrm{F}_{\mathrm{c}}$ map in green contoured at 2.5 standard deviations and the original figure-ofmerit weighted $2 \mathrm{~F}_{\mathrm{O}}-\mathrm{F}_{\mathrm{c}}$ map in blue contoured at 1.0 standard deviation obtained after initial refinement before addition of solvent or ligand to the structure factor calculations. (B) Comparison of binding modes of $\mathbf{1 5}$ (black) bound to ALDH1A1 (orange) and $\mathbf{2}$ (yellow), $\mathrm{NADH}$ (green), and $\mathrm{NAD}^{+}$(purple) bound to $\mathrm{ALDH} 2$ (cyan). NAD ${ }^{+} / \mathrm{NADH}$ binding positions obtained from PDB1O02 and 1O04. (C) Lineweaver-Burk representation of the uncompetitive inhibition pattern for 15 versus varied $\mathrm{NAD}^{+}$for $\mathrm{ALDH}^{\mathrm{A}} 1$ at saturating concentrations of propionaldehyde $(1 \mathrm{mM})$. (D) Lineweaver-Burk representation of competitive inhibition for $\mathbf{1 5}$ versus varied $\mathrm{NAD}^{+}$with $\mathrm{ALDH} 2$ at saturating concentration of propionaldehyde $(1 \mathrm{mM})$. Values are the mean/SEM of three independent experiments $(n=3)$. 

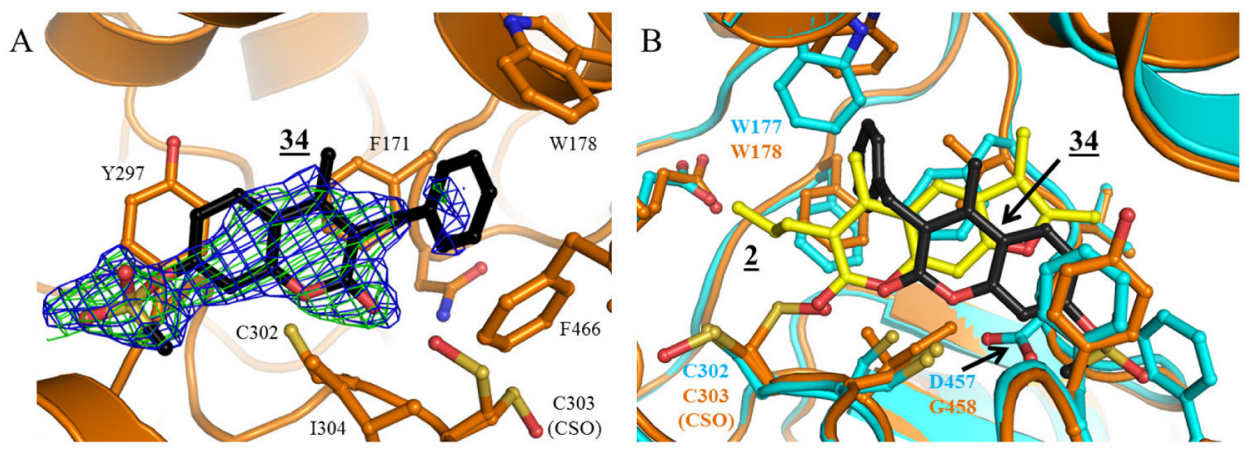

Figure 5. Binding mode of compound 34

(A) Electron density map of $\mathbf{3 4}$ bound to ALDH1A1 with the original figure-of-merit weighted $\mathrm{F}_{\mathrm{o}}-\mathrm{F}_{\mathrm{c}}$ map in green contoured at 2.5 standard deviations and the original figure-ofmerit weighted $2 \mathrm{~F}_{\mathrm{o}}-\mathrm{F}_{\mathrm{c}}$ map in blue contoured at 1.0 standard deviation obtained after initial refinement before addition of solvent or ligand to the structure factor calculation. (B) Overlay of $\mathbf{3 4}$ (black) bound to ALDH1A1 (orange) and $\mathbf{2}$ (yellow) bound to ALDH2 (cyan). 
A

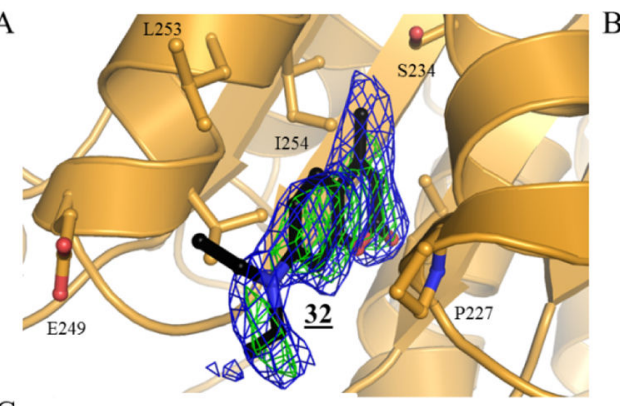

$\mathrm{C}$

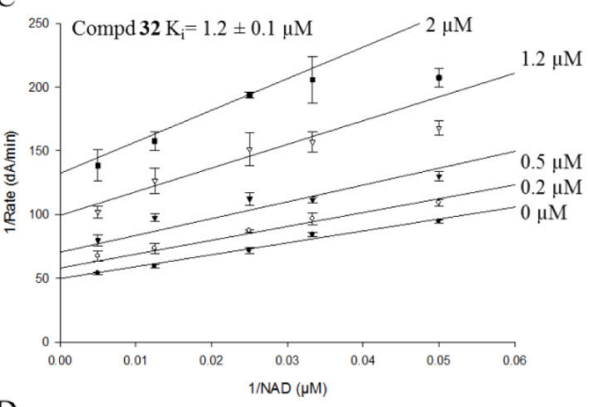

D

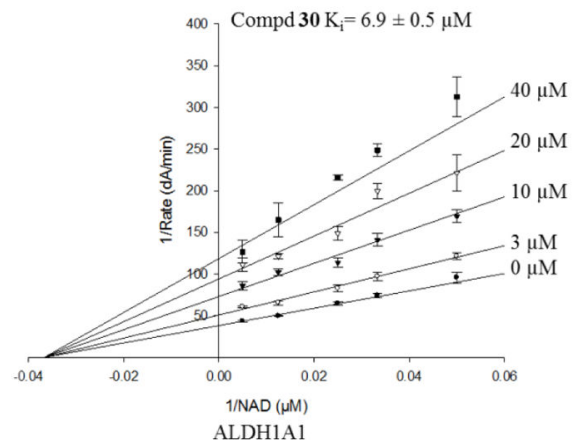

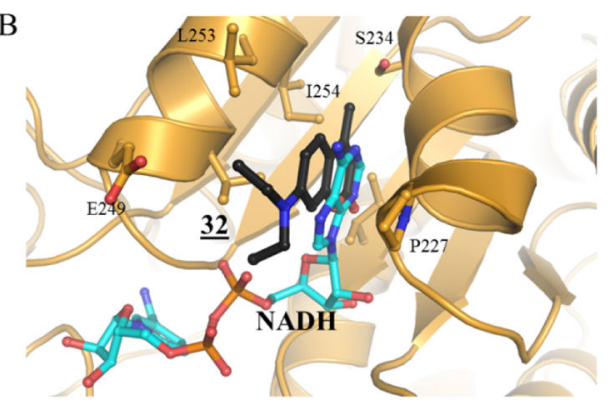
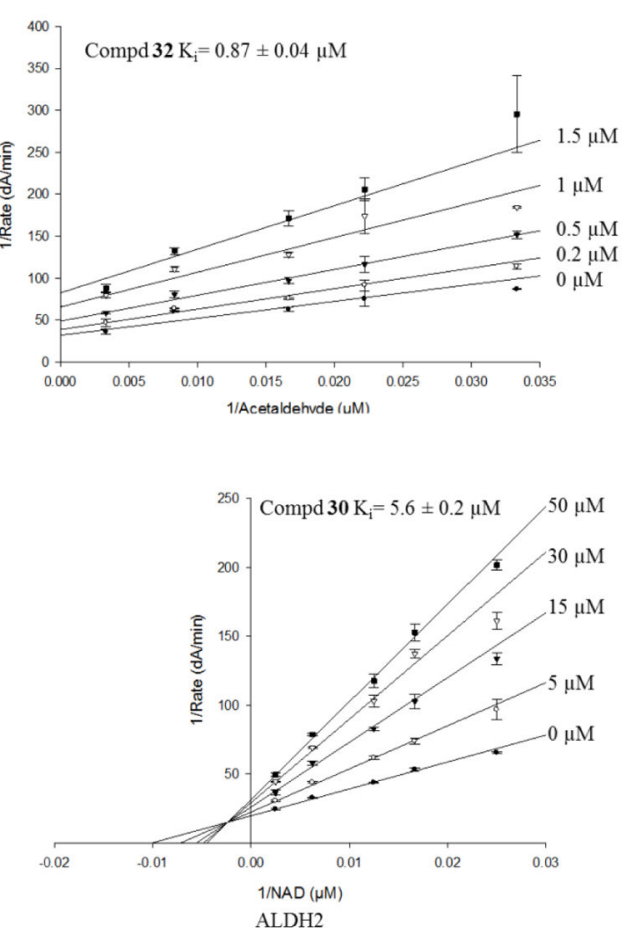

Figure 6. Binding characteristics of substituted coumarins

(A) Representative electron density map of $\mathbf{3 2}$ bound to ALDH1A1 with the original figureof-merit weighted $\mathrm{F}_{\mathrm{o}}-\mathrm{F}_{\mathrm{c}}$ map in green contoured at 2.5 standard deviations and the original figure-of-merit weighted $2 \mathrm{~F}_{\mathrm{o}}-\mathrm{F}_{\mathrm{c}}$ map in blue contoured at 1.0 standard deviation obtained after initial refinement before addition of solvent or ligand to the structure factor calculations. (B) Overlay of $\mathbf{3 2}$ and NADH (PDB 4WB9) bound to ALDH1A1 (C) Lineweaver-Burk representation of noncompetitive inhibition for $\mathbf{3 2}$ versus varied $\mathrm{NAD}^{+}$ with ALDH1A1 at saturating concentrations of propionaldehyde $(1 \mathrm{mM})$ and noncompetitive inhibition for $\mathbf{3 2}$ versus varied acetaldehyde at saturating concentrations of $\mathrm{NAD}^{+}(1 \mathrm{mM})$.(D) Lineweaver-Burk representations of noncompetitive inhibition for $\mathbf{3 0}$ versus varied $\mathrm{NAD}^{+}$with $\mathrm{ALDH} 1 \mathrm{~A} 1$ and of noncompetitive inhibition (mixed-type) inhibition for 30 with varied $\mathrm{NAD}^{+}$with $\mathrm{ALDH} 2$ at saturating concentrations of propionaldehyde $(1 \mathrm{mM})$. Values are the mean/SEM of three independent experiments $(n=3)$. 


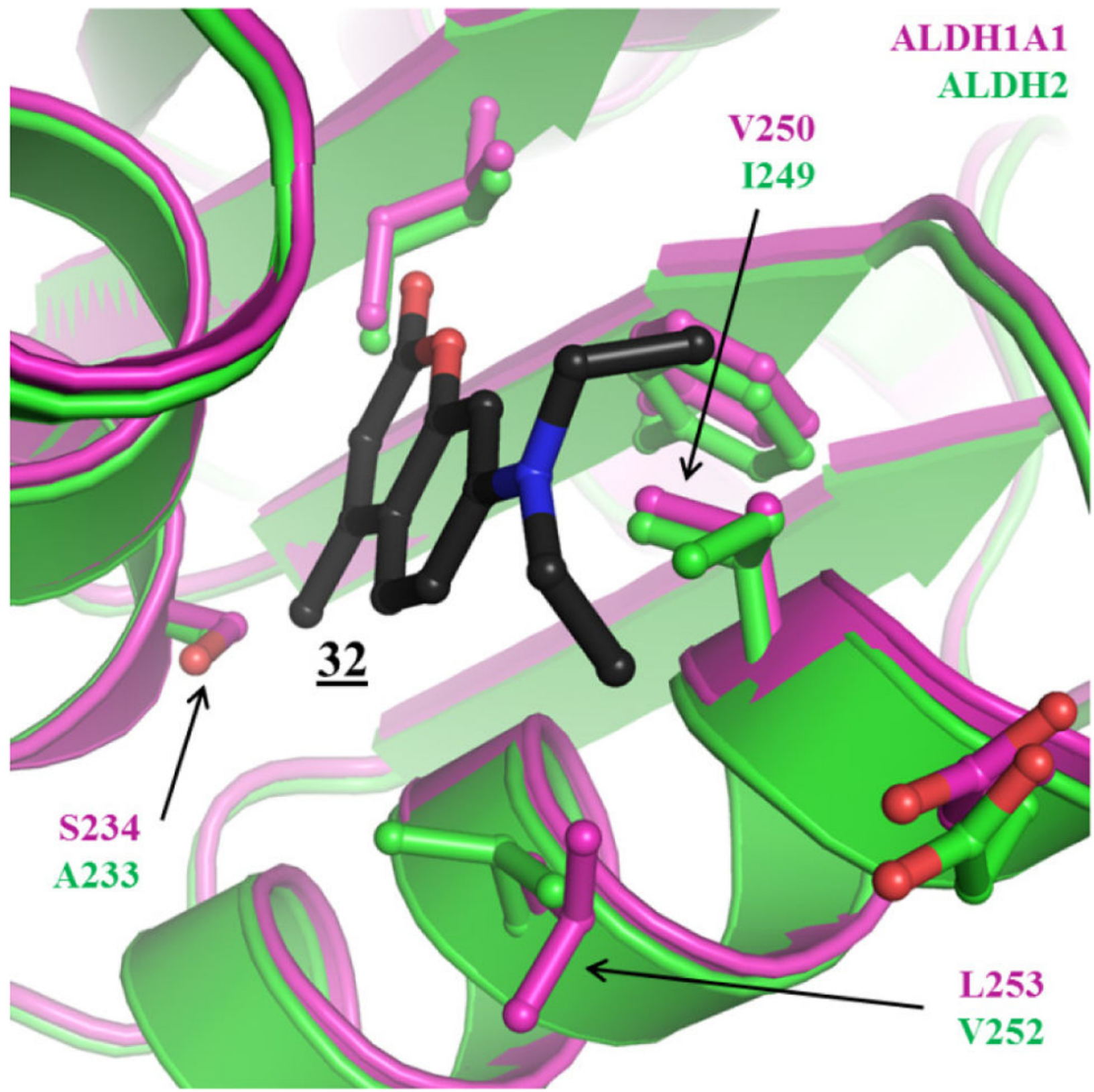

Figure 7. Selectivity for 32

Comparison of binding of $\mathbf{3 2}$ (black) to ALDH1A1 (purple) overlayed onto the equivalent region of ALDH2 (green). The non-conserved residues between ALDH1A1 and ALDH2 are labeled. 


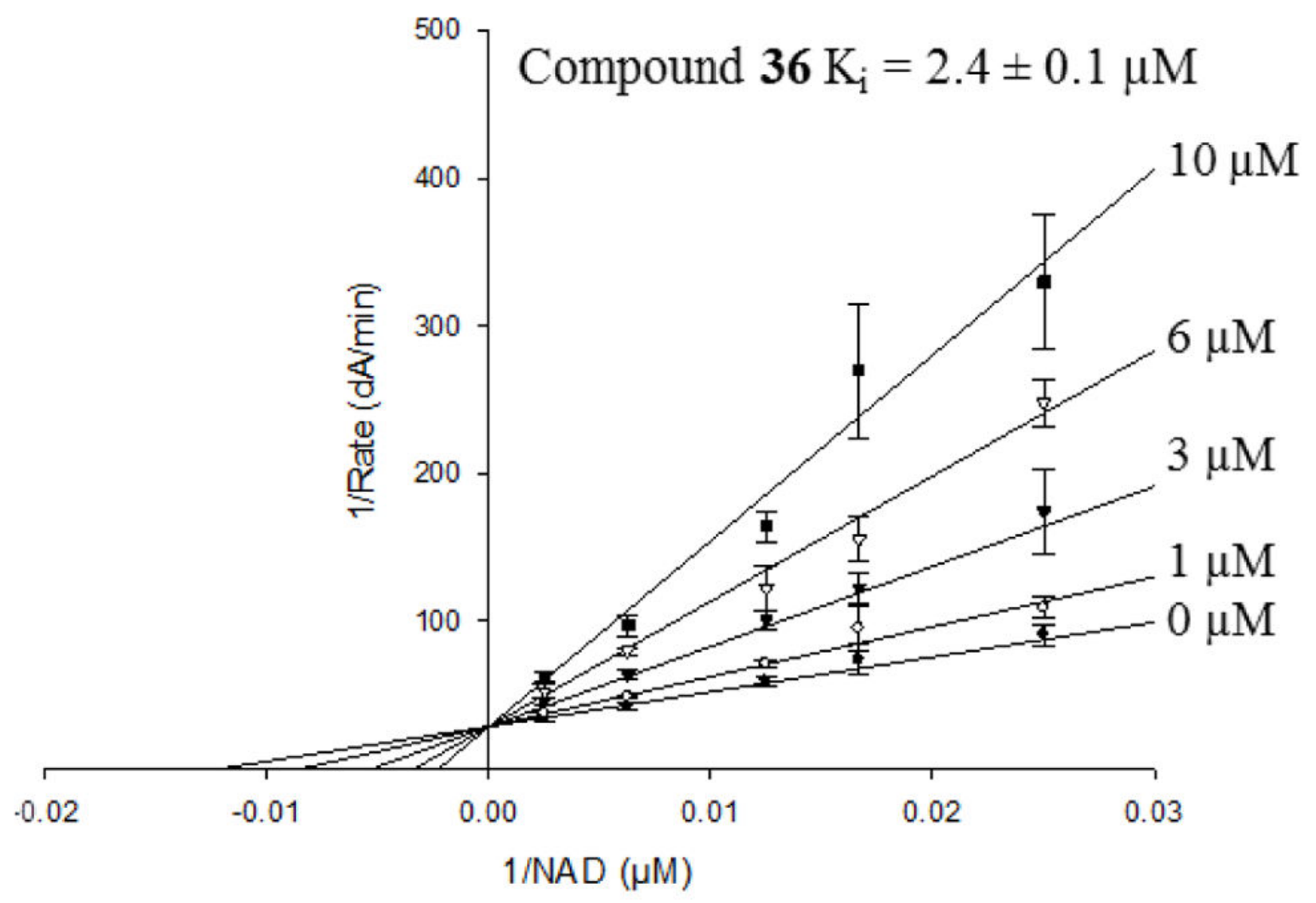

Figure 8.

Lineweaver-Burk representation of competitive inhibition for $\mathbf{3 6}$ versus varied $\mathrm{NAD}^{+}$with ALDH2 at saturating concentrations of propionaldehyde $(1 \mathrm{mM})$. Values are the mean/SEM of three independent experiments $(n=3)$ 


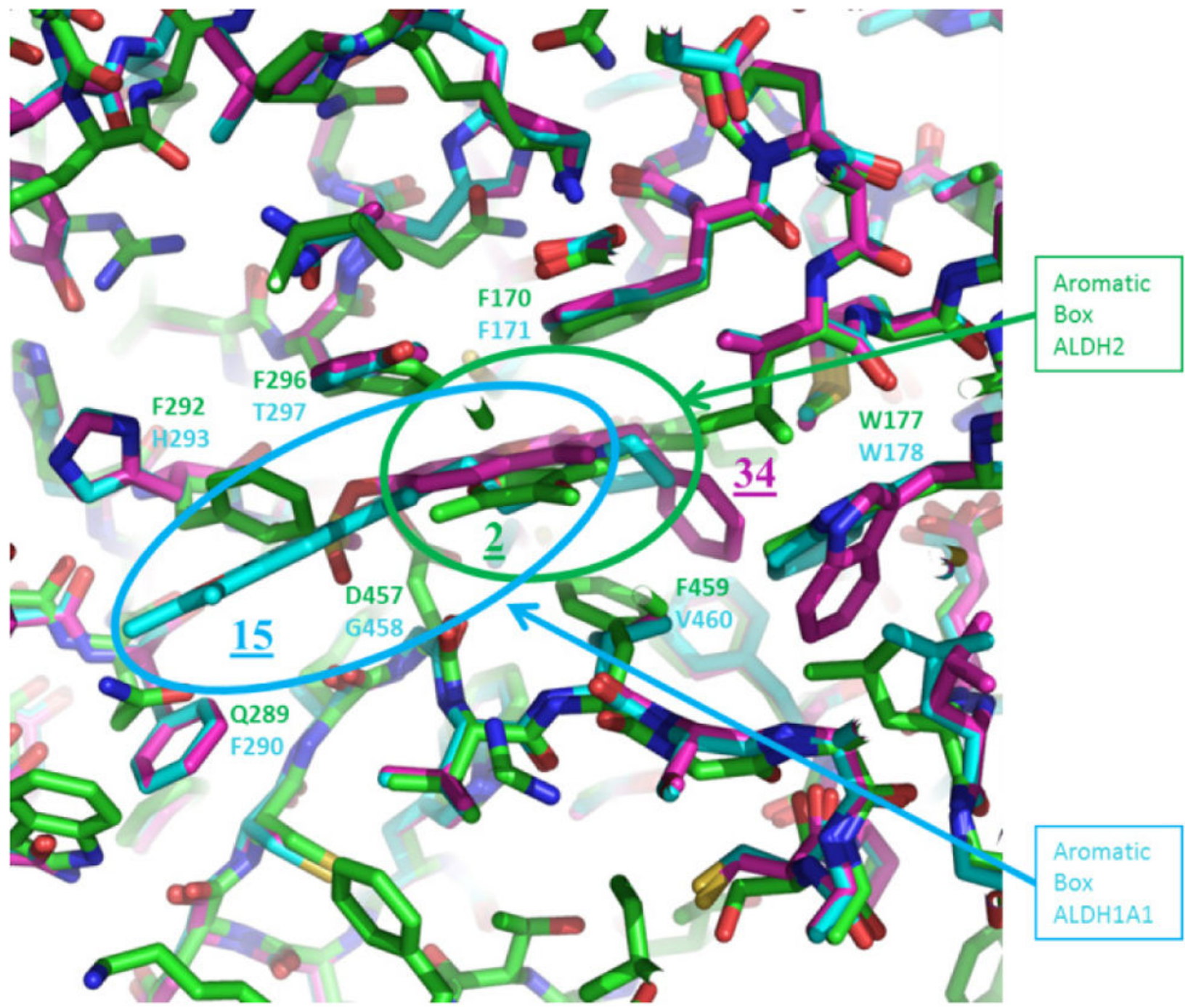

Figure 9. Distinct aromatic binding boxes of ALDH1A1 and ALDH2

Compound 2 bound to ALDH2 shown in green. Compound 15 bound to ALDH1A1 shown in cyan. Compound 34 bound to ALDH1A1 shown in purple. 


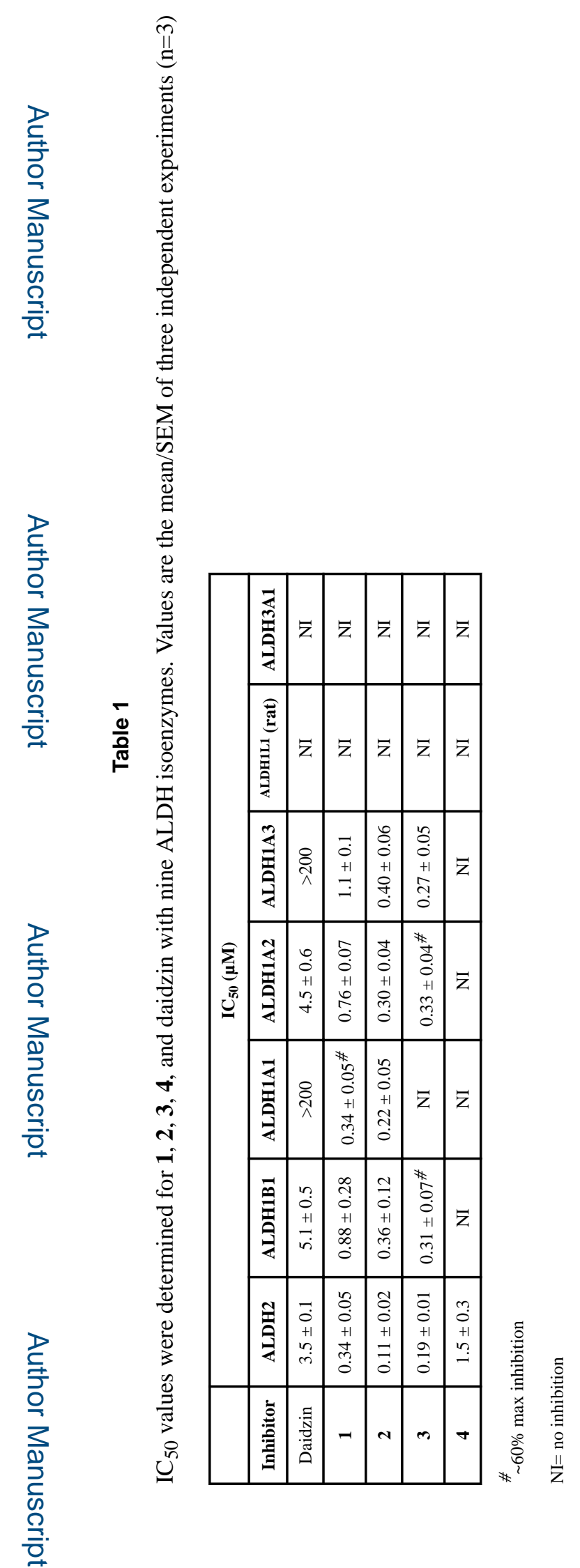

J Med Chem. Author manuscript; available in PMC 2018 January 12. 
Table 2

Data collection and refinement statistics for enzyme-compound complexes

\begin{tabular}{|c|c|c|c|c|}
\hline Compound & 2 & 15 & 34 & 32 \\
\hline Enzyme & ALDH2 & ALDH1A1 & ALDH1A1 & ALDH1A1 \\
\hline PDB Code & $5 \mathrm{~L} 13$ & $5 \mathrm{~L} 2 \mathrm{M}$ & $5 \mathrm{~L} 2 \mathrm{~N}$ & $5 \mathrm{~L} 2 \mathrm{O}$ \\
\hline \multicolumn{5}{|l|}{ Data Collection } \\
\hline Date of Collection & Nov 2012 & Mar 2016 & Mar 2016 & Mar 2016 \\
\hline Space Group & $\mathrm{P} 2_{1} 2_{1} 2_{1}$ & P422 & $\mathrm{P} 422$ & $\mathrm{P} 1$ \\
\hline \multicolumn{5}{|l|}{ Cell Dimensions } \\
\hline $\mathrm{a}, \mathrm{b}, \mathrm{c}(\AA)$ & $99,127,295$ & $109,109,83$ & $109,109,83$ & $91,98,127$ \\
\hline$a, \beta, \gamma(\operatorname{deg})$ & $90,90,90$ & $90,90,90$ & $90,90,90$ & $81,86,64$ \\
\hline Resolution ( $(̊)$ & $50.0-2.40$ & $50.0-1.70$ & $50.0-1.70$ & $50.0-2.05$ \\
\hline $\mathrm{R}_{\text {merge }}$ & $0.093(0.280)^{a}$ & $0.077(0.501)$ & $0.099(0.654)$ & $0.069(0.280)$ \\
\hline $\mathrm{R}_{\text {meas }}$ & $\mathrm{ND}^{\mathrm{b}}$ & $0.083(0.546)$ & $0.108(0.716)$ & $0.093(0.377)$ \\
\hline $\mathrm{R}_{\mathrm{pim}}$ & $\mathrm{ND}^{\mathrm{b}}$ & $0.032(0.214)$ & $0.041(0.284)$ & $0.061(0.251)$ \\
\hline $\mathrm{CC} 1 / 2$ & $\mathrm{ND}^{\mathrm{b}}$ & $0.943(0.778)$ & $0.905(0.637)$ & $0.955(0.845)$ \\
\hline$I / \sigma_{\langle I\rangle}$ & $16.2(5.0)$ & $19.3(3.6)$ & $14.2(2.8)$ & $10.7(2.7)$ \\
\hline Completeness (\%) & $94.2(85.7)$ & $97.6(97.0)$ & $98.9(100)$ & $90.8(91.7)$ \\
\hline Redundancy & $5.0(4.8)$ & $6.4(6.3)$ & $6.4(6.1)$ & $2.1(2.1)$ \\
\hline \multicolumn{5}{|l|}{ Refinement } \\
\hline No. of reflections & 131537 & 51675 & 52057 & 211433 \\
\hline No. of protein atoms & 30485 & 3811 & 3798 & 30488 \\
\hline No. of water molecules & 1711 & 309 & 283 & 2326 \\
\hline No. of inhibitor molecules & 8 & 1 & 1 & 8 \\
\hline Occupancy of inhibitor(s) & 1 & 1 & 1 & 1 \\
\hline $\mathrm{R}_{\mathrm{work}} / \mathrm{R}_{\text {free }}$ & $0.16 / 0.21$ & $0.22 / 0.24$ & $0.21 / 0.24$ & $0.17 / 0.20$ \\
\hline \multicolumn{5}{|l|}{ RMSD } \\
\hline Bond Length $(\AA)$ & 0.009 & 0.010 & 0.010 & 0.012 \\
\hline Bond Angle (deg) & 1.324 & 1.395 & 1.373 & 1.388 \\
\hline \multicolumn{5}{|l|}{ Ramachandran $\operatorname{plot}^{c}$} \\
\hline Preferred (\%) & $96.05 \%$ & $97.14 \%$ & $97.34 \%$ & $97.05 \%$ \\
\hline Outliers (\%) & $0.56 \%$ & $0.20 \%$ & $0.20 \%$ & $0.20 \%$ \\
\hline Clashscore (percentile) ${ }^{\mathcal{C}}$ & $1.69(100 \%)$ & $2.76(99 \%)$ & $1.98(99 \%)$ & $1.70(100 \%)$ \\
\hline MolProbity score (percentile) ${ }^{c}$ & $1.16(100 \%)$ & $1.22(98 \%)$ & $1.09(99 \%)$ & $1.23(100 \%)$ \\
\hline \multicolumn{5}{|l|}{ Average B $(\AA)$} \\
\hline \multirow[t]{3}{*}{ Protein } & 27.5 & 38.3 & 40.5 & 19.5 \\
\hline & Chain A: 26.4 & & & Chain A: 28.8 \\
\hline & B: 33.4 C: 29.2 & & & B: 30.3 C: 24.8 \\
\hline \multirow[t]{3}{*}{ Inhibitor } & D: $27.2 \mathrm{E}: 28.0$ & 29.7 & 38.0 & D: 23.9 E: 33.9 \\
\hline & F: $39.2 \mathrm{G}: 34.2$ & & & F: $31.0 \mathrm{G}: 26.3$ \\
\hline & H: 28.3 & & & H: 33.9 \\
\hline
\end{tabular}




\begin{tabular}{lcccc}
\hline Compound & $\mathbf{2}$ & $\mathbf{1 5}$ & $\mathbf{3 4}$ & $\mathbf{3 2}$ \\
Solvent & 29.4 & 30.2 & 35.1 & 26.5 \\
\hline
\end{tabular}

${ }^{a}$ Values in parenthesis are those from the highest resolution shell.

$\mathrm{ND}^{\mathrm{b}}$ Processing values not available in HKL2000 version 0.96

${ }^{c}$ Generated with Molprobity. 


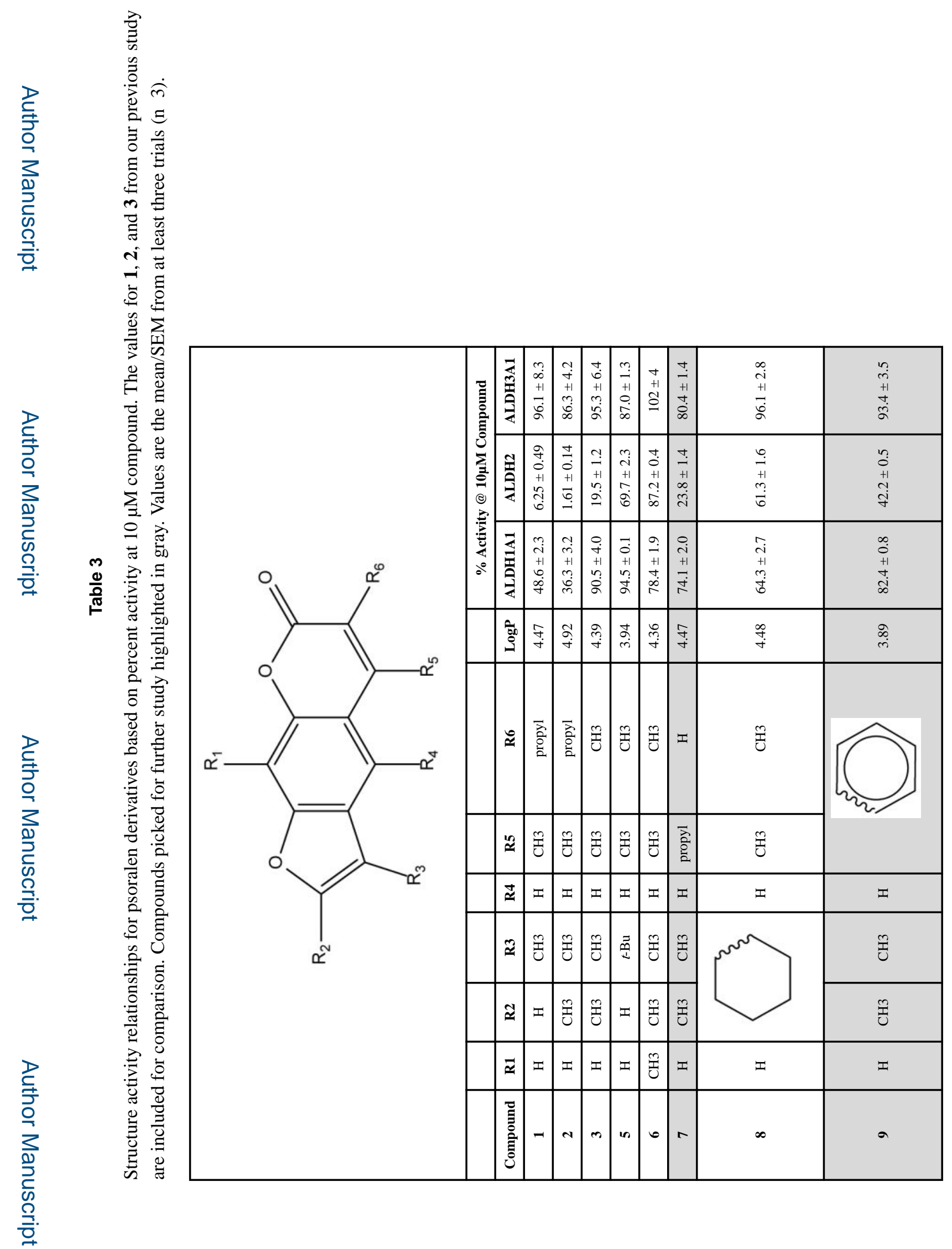

JMed Chem. Author manuscript; available in PMC 2018 January 12. 


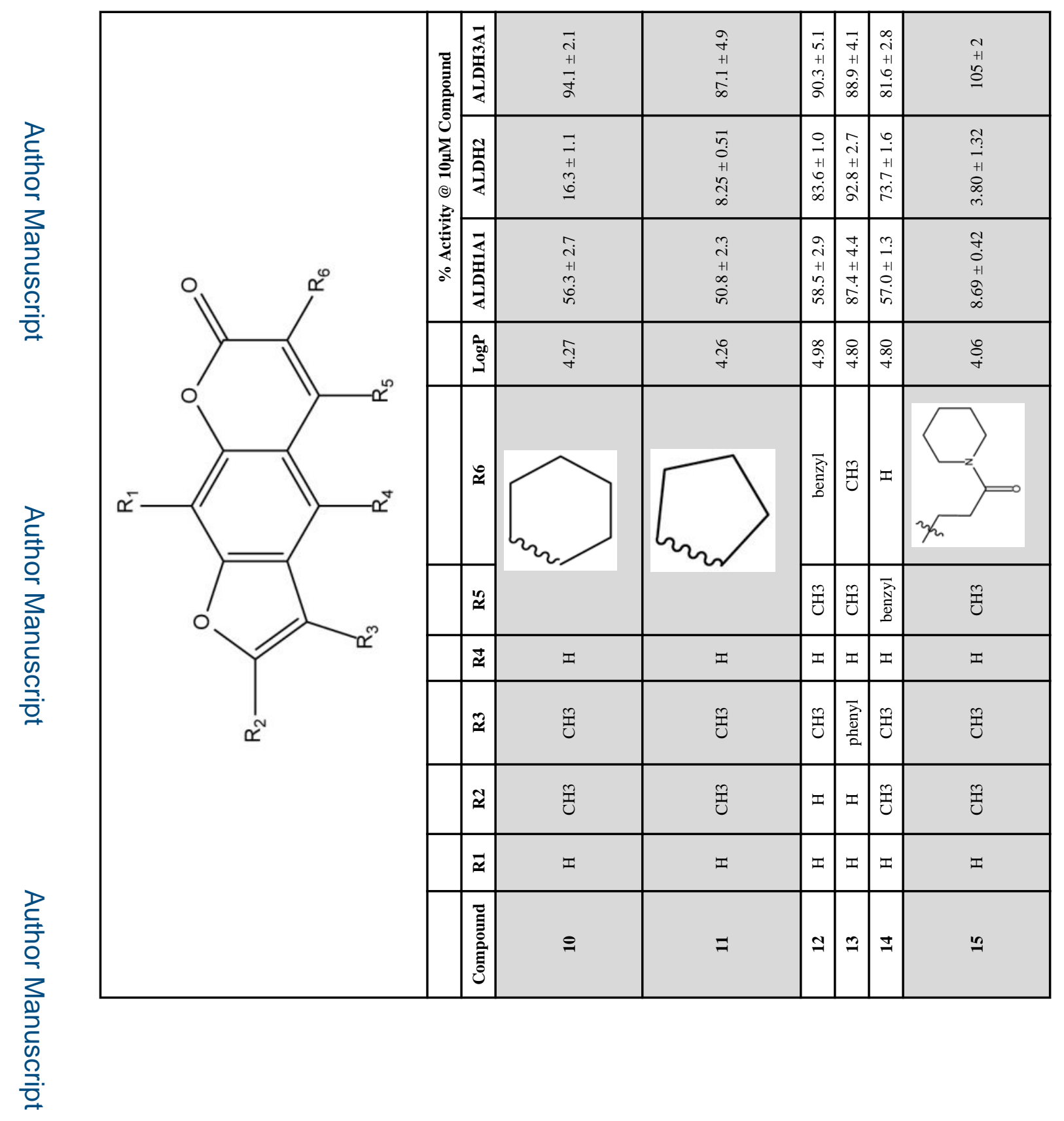

로을 


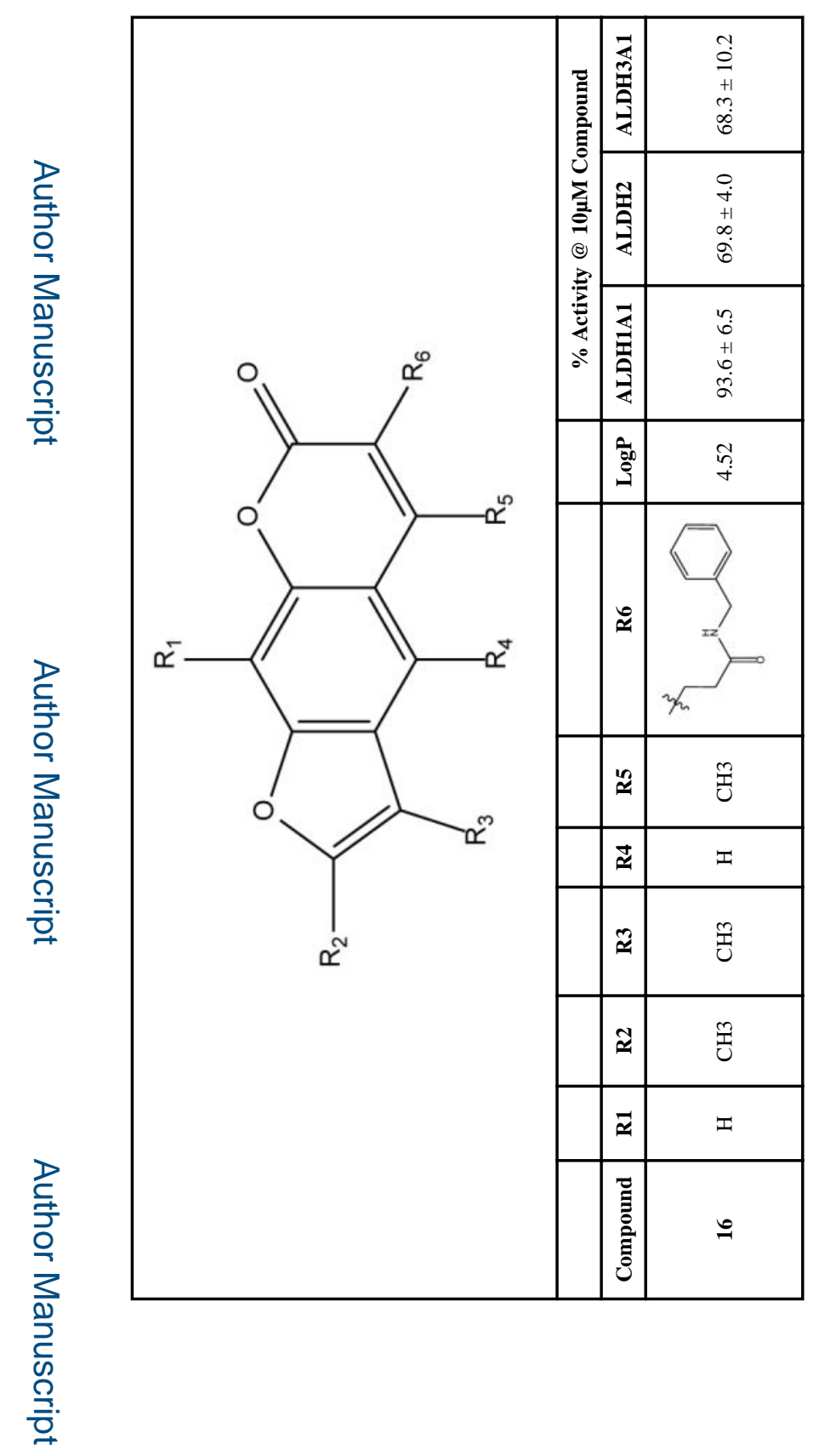

로을

J Med Chem. Author manuscript; available in PMC 2018 January 12. 


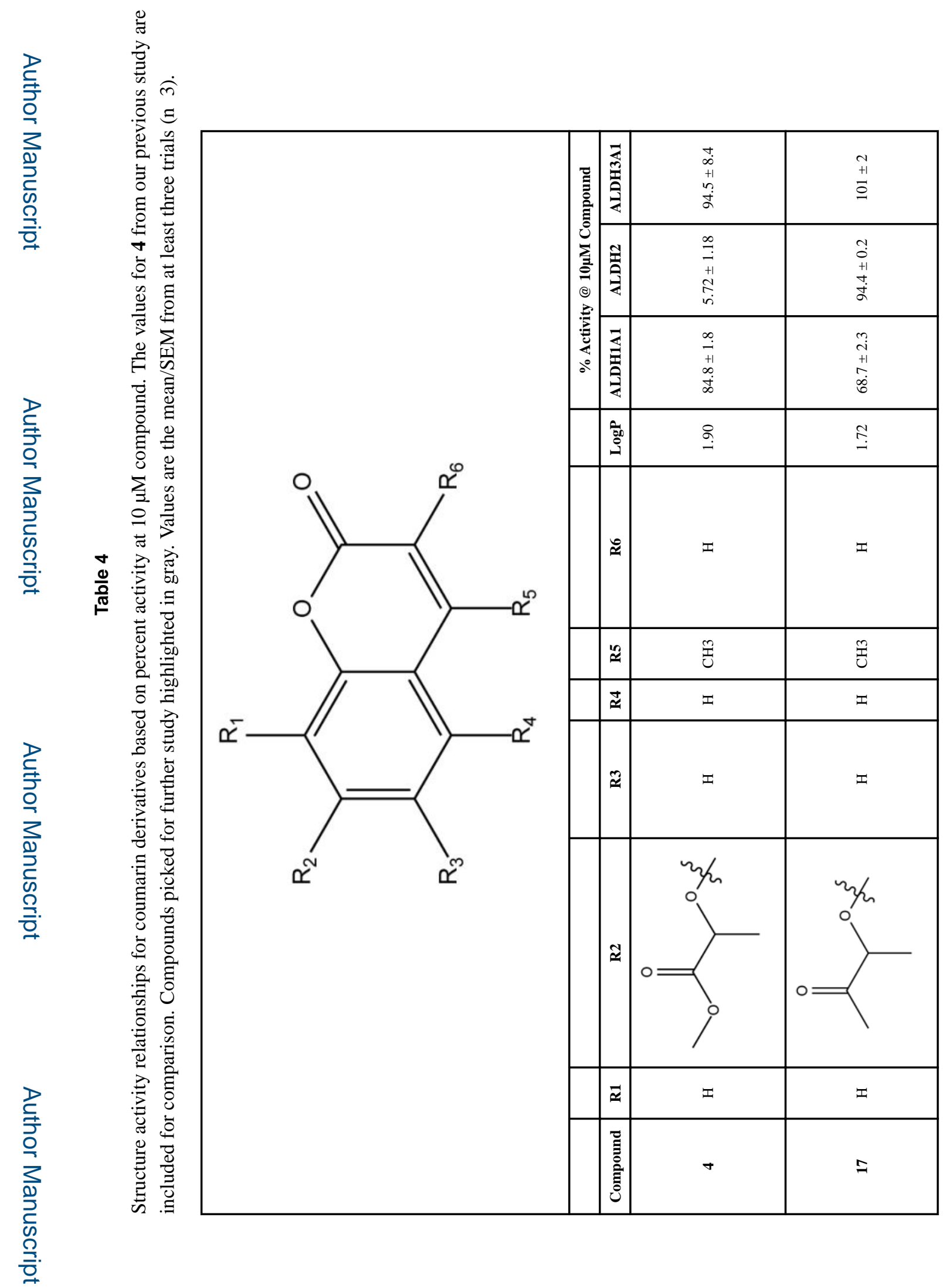

J Med Chem. Author manuscript; available in PMC 2018 January 12. 


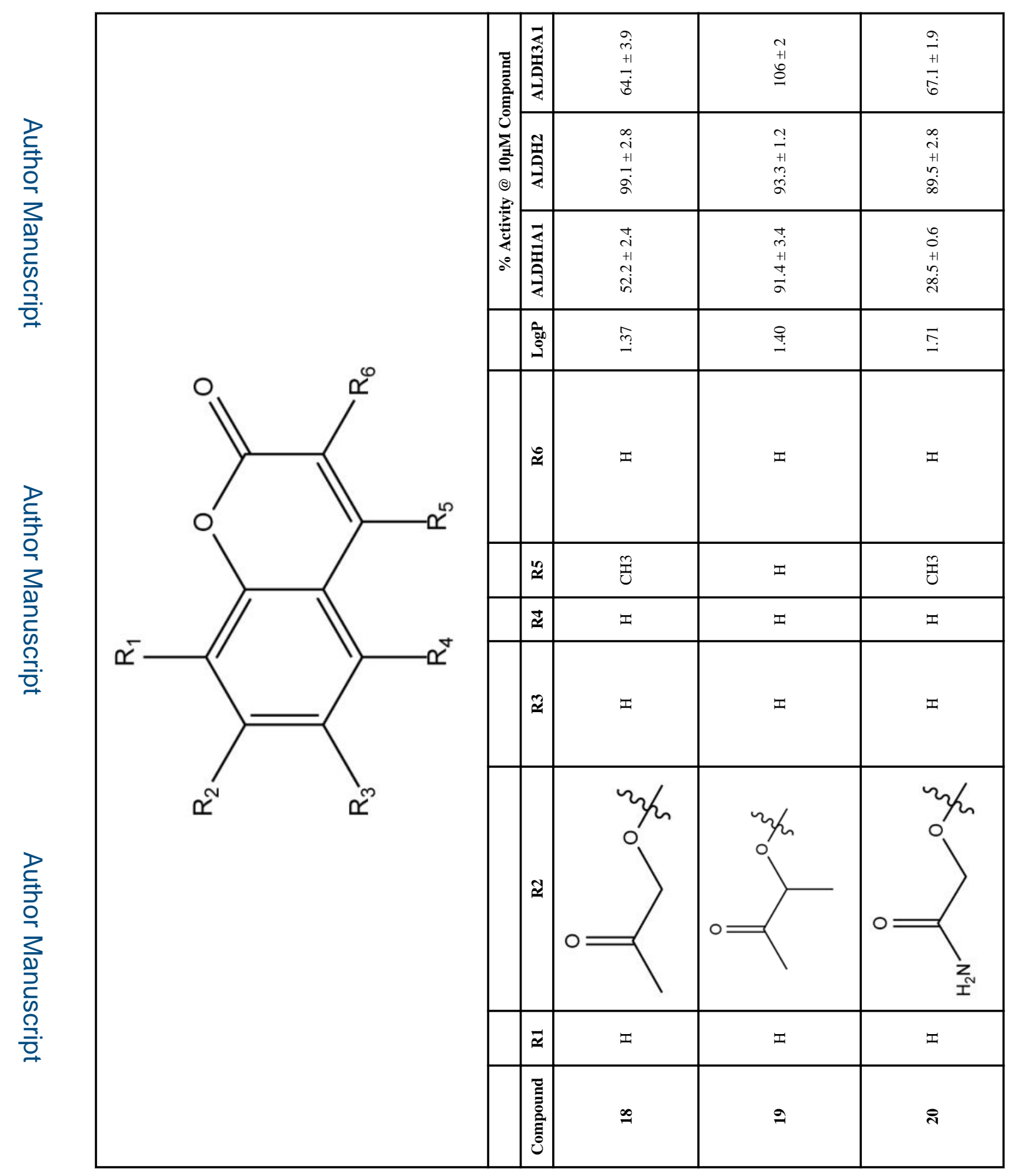

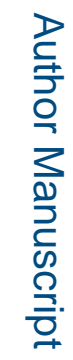




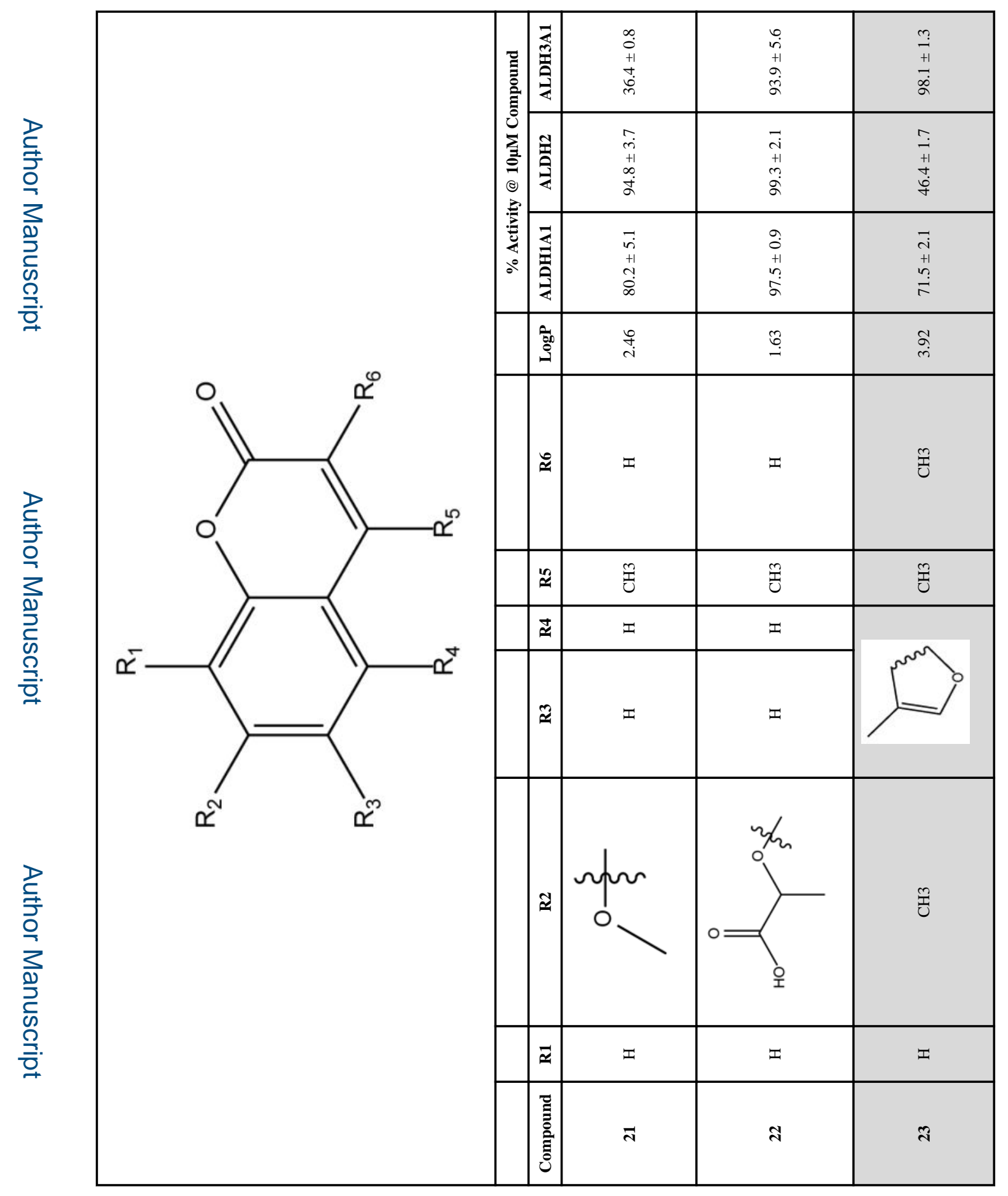

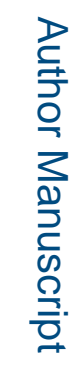




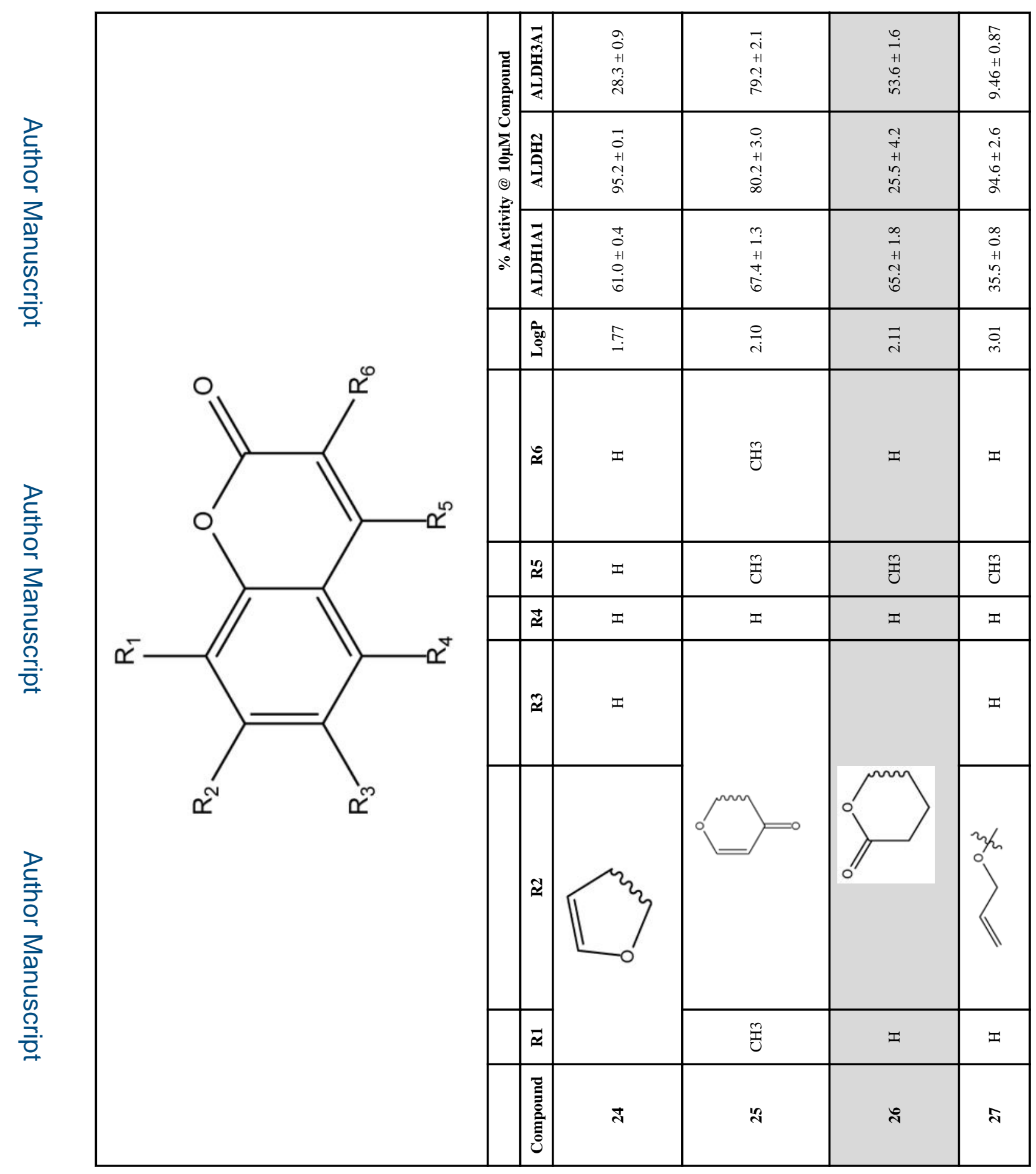

로을 


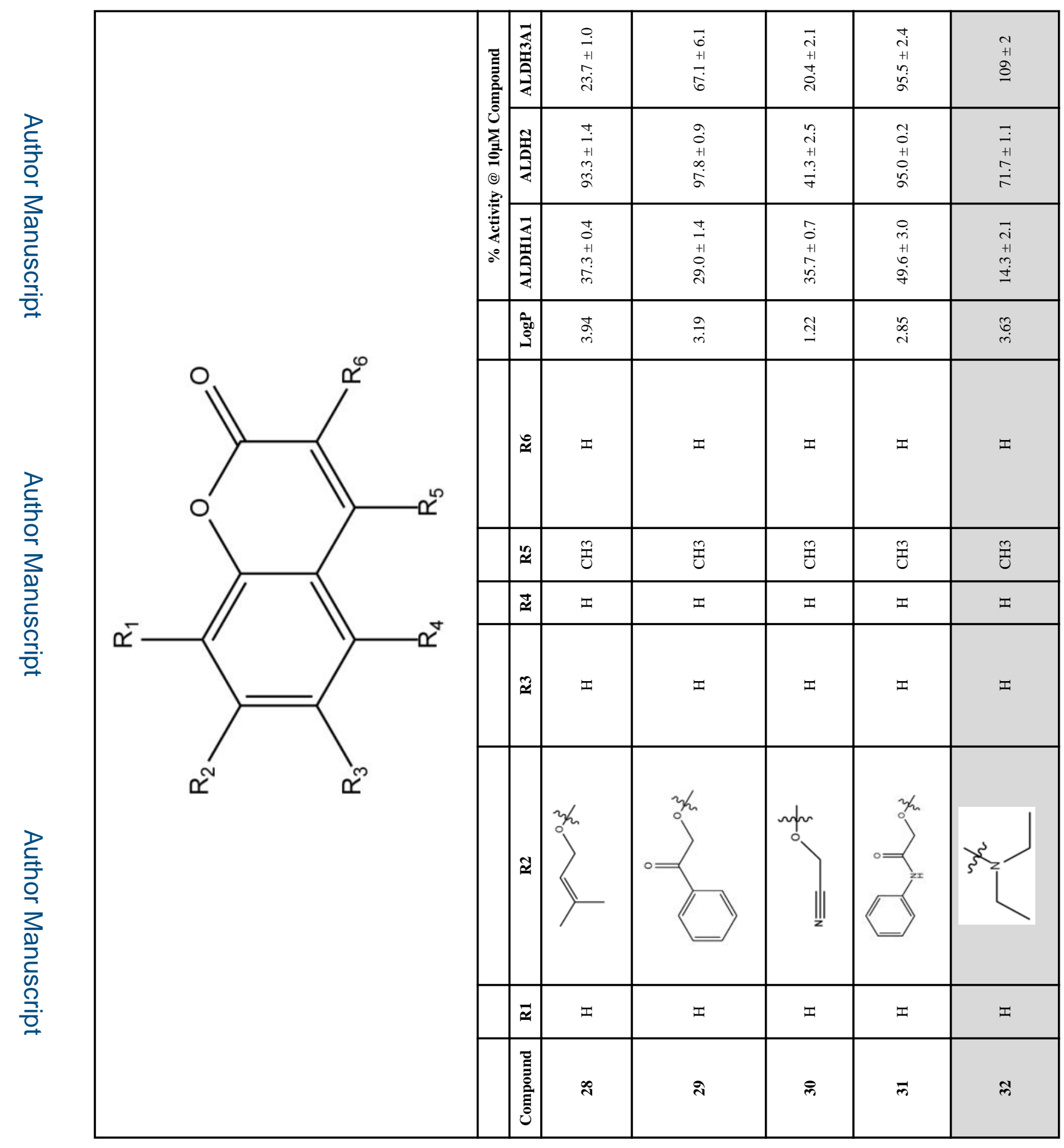

로을 


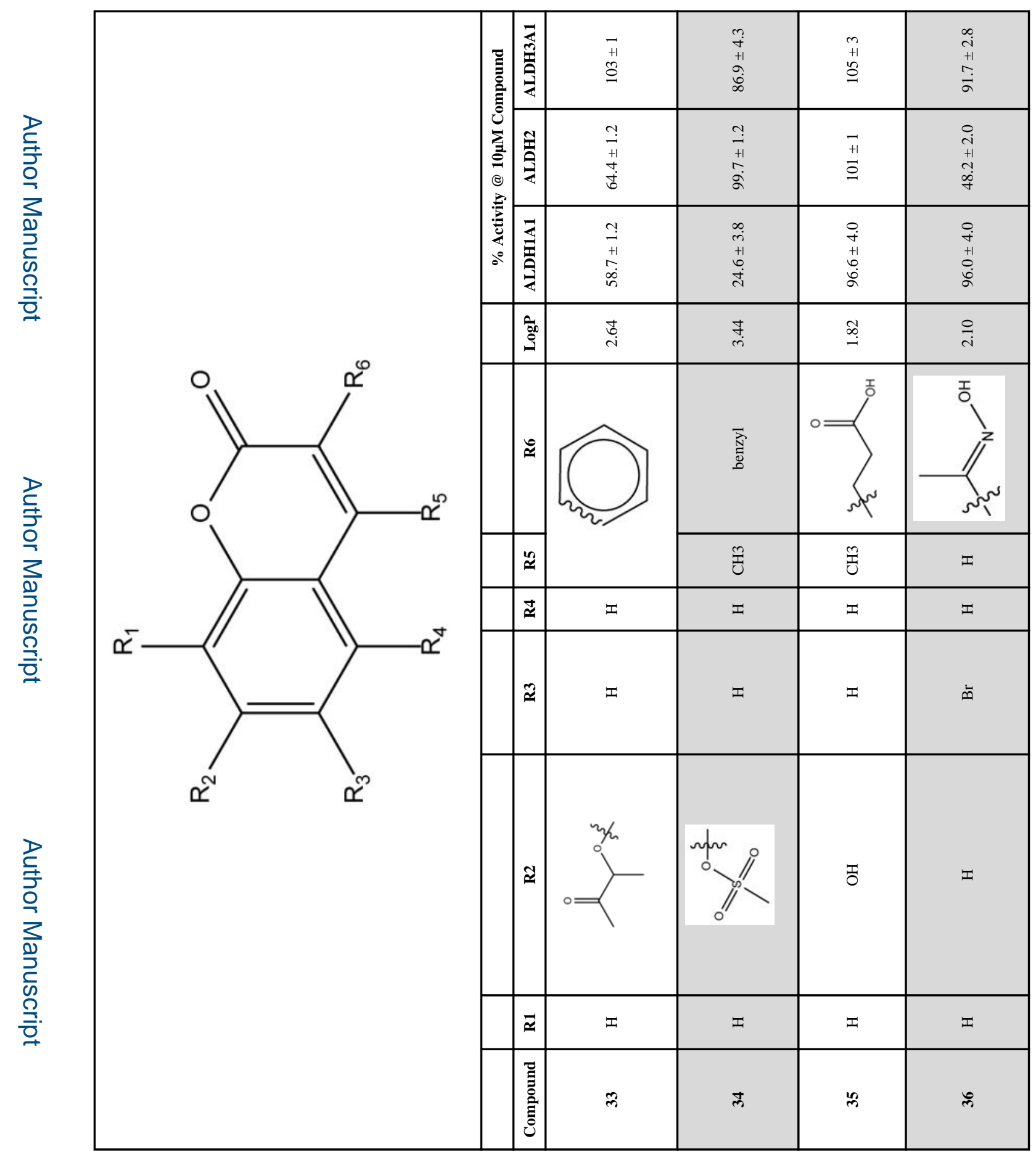

로을 


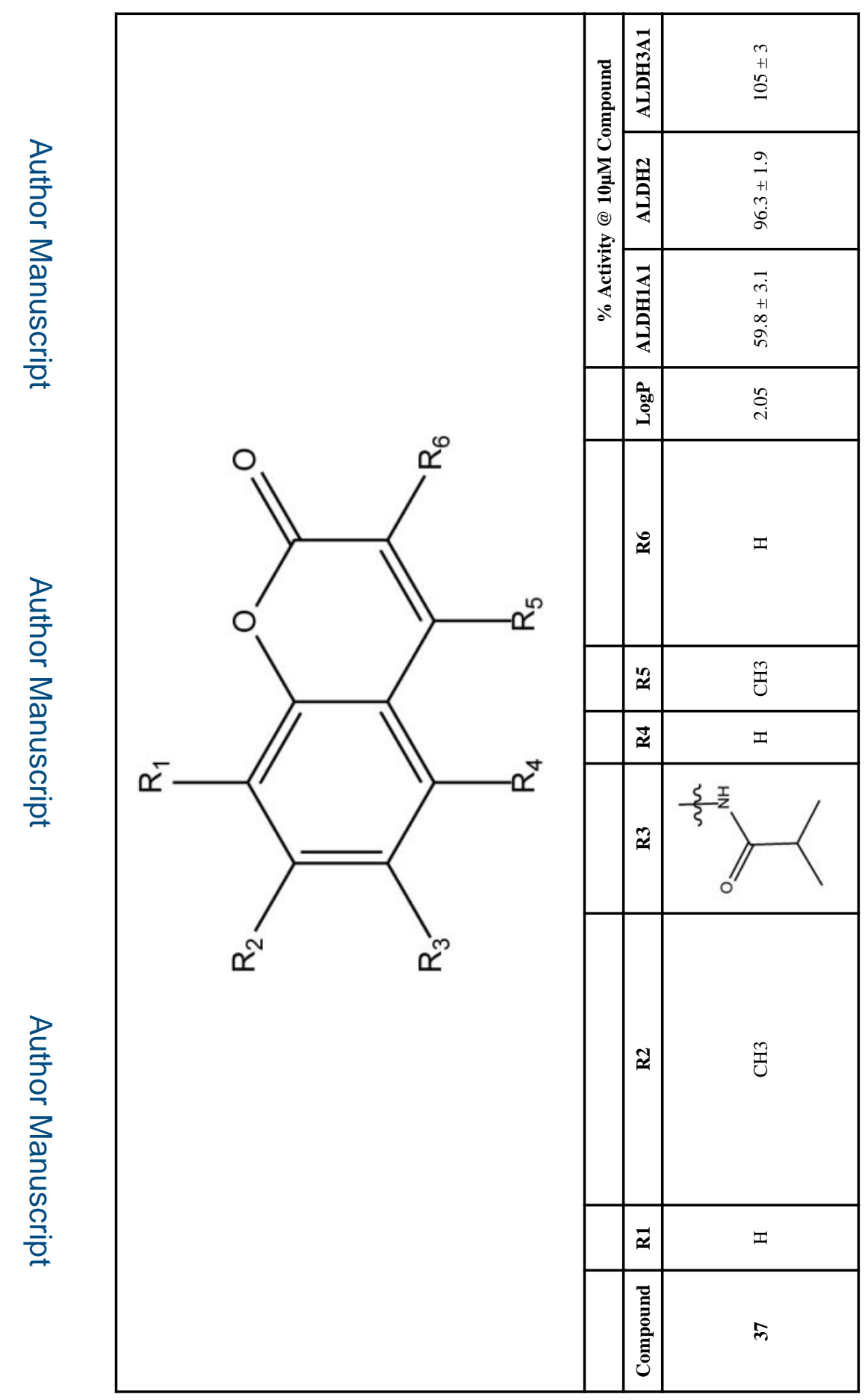

로을 


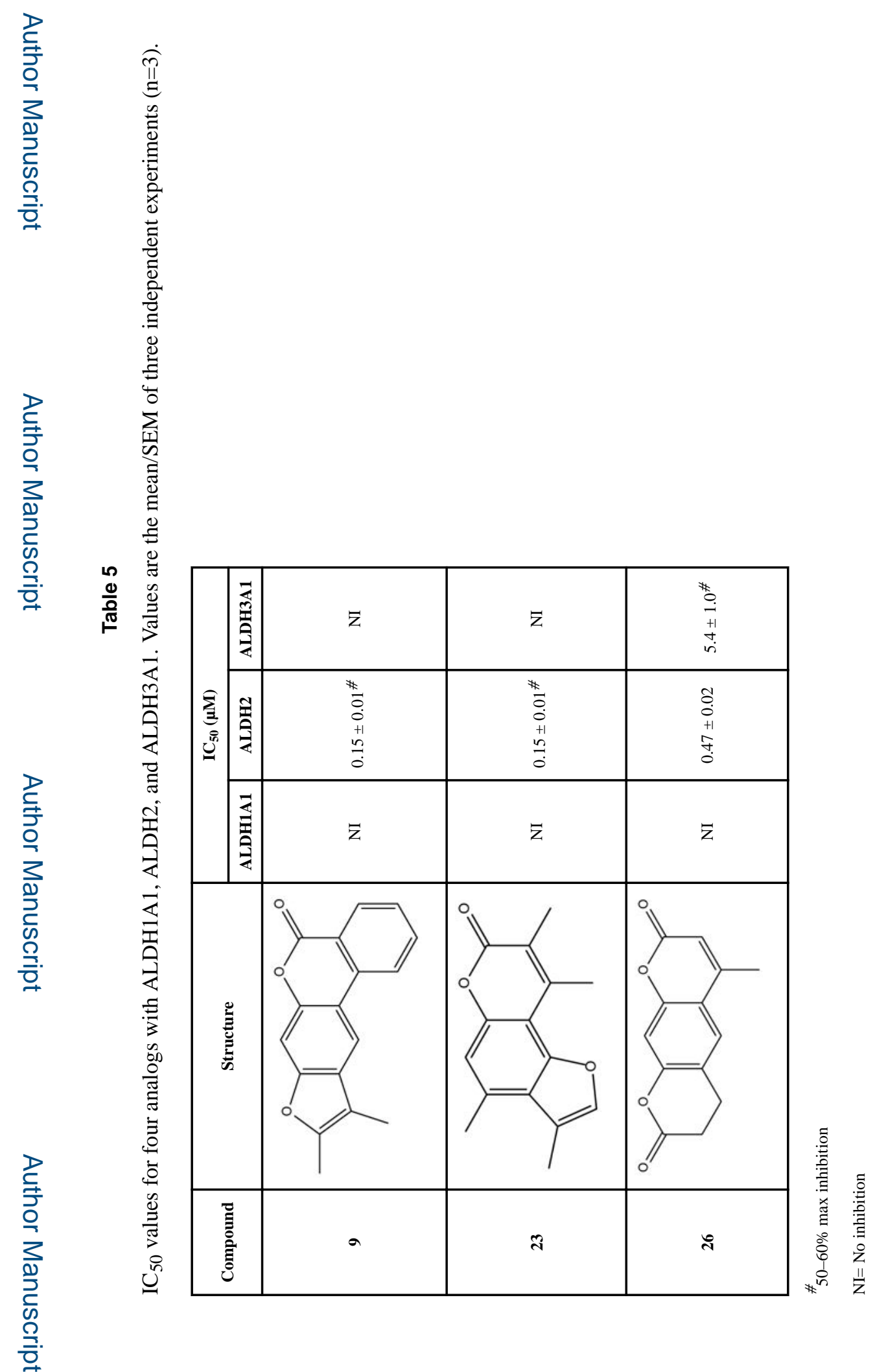

J Med Chem. Author manuscript; available in PMC 2018 January 12. 


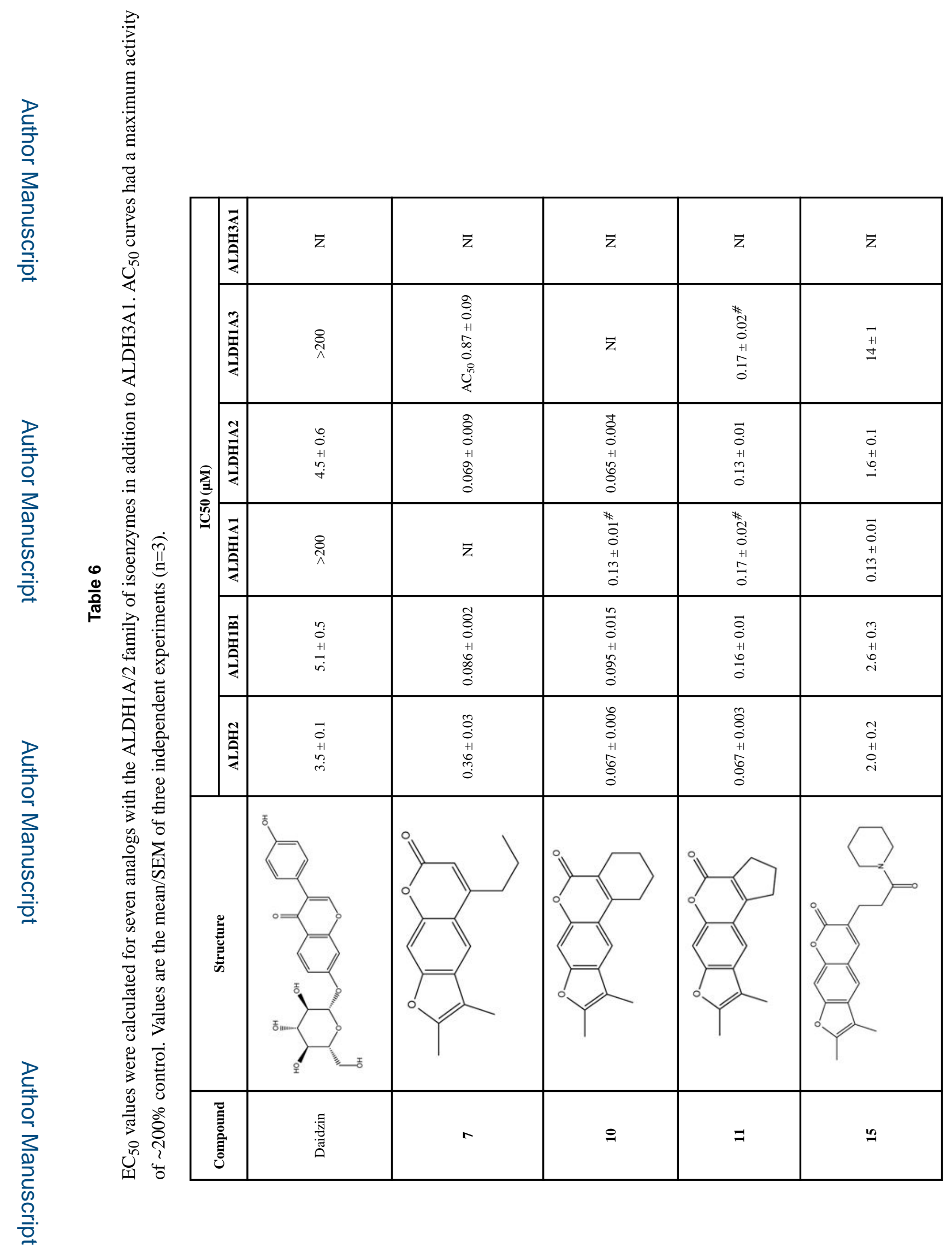

J Med Chem. Author manuscript; available in PMC 2018 January 12. 


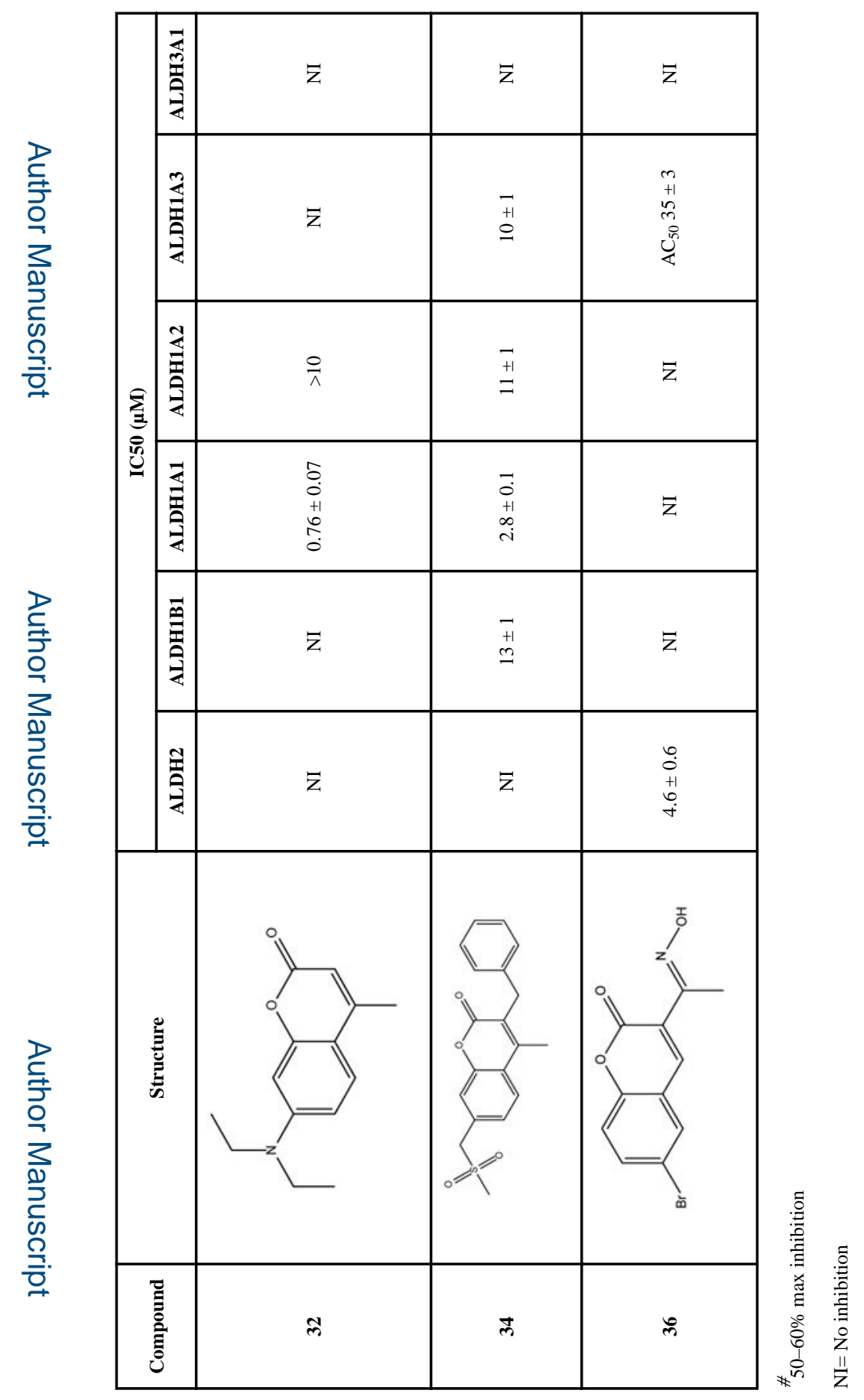

로을 